\title{
Betulinic Acid Inhibits ROS-Mediated Pyroptosis in Spinal Cord Injury by Augmenting Autophagy via the AMPK-mTOR-TFEB Signaling Pathway
}

\section{Chenyu Wu}

Wenzhou Medical University

Huanwen Chen

university of maryland shool

Rong Zhuang

Wenzhou Medical University

Yongli Wang

Huzhou Central Hospital

Xinli Hu

wenzhou medical university

Haojie Zhang

Wenzhou Medical University

Yu Xu

Wenzhou Medical University

Jiafeng Li

wenzhou medical univeristy

Yao Li

Wenzhou Medical University

Huazi Xu

Wenzhou Medical University

Hui Xu

Wenzhou Medical College - Chashan Campus: Wenzhou Medical University

Wenfei Ni

Wenzhou Medical University

kailiang zhou ( $\nabla$ zhoukailiang@wmu.edu.cn)

Wenzhou Medical University

\section{Research}

Keywords: Betulinic acid, autophagy, ROS, pyroptosis, spinal cord injury 
Posted Date: November 4th, 2020

DOI: https://doi.org/10.21203/rs.3.rs-100352/v1

License: (c) (i) This work is licensed under a Creative Commons Attribution 4.0 International License. Read Full License 


\section{Abstract}

Background冈Spinal cord injury (SCI) results in a wide range of disabilities. Its complex pathophysiological process limits the effectiveness of many clinical treatments. Betulinic acid (BA) has been shown to be an effective treatment for some neurological diseases, but it has not been studied in $\mathrm{SCl}$. In this study, we assessed the role of $\mathrm{BA}$ in $\mathrm{SCl}$ and investigated its underlying mechanism.

Methods खUsing a mouse model of $\mathrm{SCl}$, survival and functional outcomes following injury were assessed. Western blotting, ELISA, and immunofluorescence techniques were employed to analyze levels of autophagy, mitophagy, and pyroptosis; ROS- and AMPK-related signaling pathways were also examined.

Results $\bowtie$ Our results showed that BA significantly improves functional recovery following SCl. Furthermore, autophagy, mitophagy, ROS-activity and pyroptosis were implicated in the mechanism of BA in the treatment of SCl. Specifically, our results suggest that BA restored autophagy flux following injury, which induces mitophagy to eliminate the accumulation of ROS and subsequently inhibits pyroptosis. Further mechanistic studies revealed that BA likely regulates autophagy and mitophagy via the AMPKmTOR-TFEB signaling pathway.

Conclusion: $\mathrm{BA}$ can significantly promote the recovery following $\mathrm{SCl}$ and that it may be a promising therapy for SCl.

\section{Introduction}

Spinal cord injury $(\mathrm{SCl})$ is a disabling disease that often results in significant motor and sensory dysfunctions [1], and it affects over 500,000 patients in the United States alone [2]. Currently, there are only a few therapeutic interventions for $\mathrm{SCl}$, with high-dose methylprednisolone [3], ganglioside [4], immunoglobulin G [5], and exogenous basic fibroblast growth factor [6] showing some clinical benefit in select patients; however, these agents have largely been unable to improve recovery following SCI. The dearth of therapeutic options for $\mathrm{SCl}$ is likely due to its complex pathophysiology. In brief, $\mathrm{SCl}$ is thought to involve two distinct injury mechanisms: primary and secondary. Primary injury occurs as a direct consequence of mechanical damage of the spinal cord, whereas secondary injury is triggered by a delayed sequence of complex biochemical and cellular processes such as oxidative stress accumulation, inflammation [7], apoptosis, programmed cell death, and various other processes [8]. Together, primary and secondary injuries lead to irreversible neuronal damage, which ultimately culminates in poor functional recovery of patients following SCI [9]. Thus, preventing irreversible damage by targeting pathways of programmed cell death such as apoptosis, pyroptosis, and autophagy may be a promising strategy for treating $\mathrm{SCl}[10-12]$.

Pyroptosis, an inflammatory form of programmed cell death, is known to play a detrimental role in acute central nervous system (CNS) injuries such as traumatic brain injury, cerebral ischemia, and SCI [13-15]. Morphologically, pyroptosis is characterized by cell swelling, rupture, and release of cytoplasmic contents [16]. Mechanistically, following CNS injury, inflammasomes (such as NLRP3, ASC, Caspase-1) are 
activated to cleave GSDMD and release proinflammatory cytokines (interleukin-1 $\beta$ and 18 ), ultimately leading to pyroptosis [16-18]. In addition, extensive studies have demonstrated that a series of pathological changes such as hemorrhage, hypoxia, and edema after $\mathrm{SCl}$ can cause accumulations of reactive oxygen species (ROS), which can act as secondary messengers to induce NLRP3 inflammasomemediated pyroptosis $[19,20]$.

Autophagy, key cellular process for removing cytosolic waste and damaged organelles [21,22], plays a key role in the pathophysiology of neurological diseases [23]. Overall, activation of autophagy is considered protective in $\mathrm{SCl}$, which can attenuate pyroptosis [24] and improve functional recovery [25]. More specifically, increased autophagy stimulates mitophagy ( autophagic degradation of damaged mitochondria), restricts the production of ROS, dampen the activation of NLRP3 inflammasomes, and suppress the secretion of IL-1 $\beta$ and IL-18, ultimately inhibiting pyroptosis [22, 26, 27]. Therefore, autophagy augmentation may be a viable strategy to suppress pyroptosis and improve functional recovery following $\mathrm{SCl}$.

Betulinic acid (BA), a natural pentacyclic triterpenoid, is known to be found in Chinese herbal medicine extensively [28]. More importantly, BA has the ability to cross the blood-brain barrier, which is of great significance in the treatment of CNS pathologies [29,30]. Past studies have shown that BA exerts neuroprotective effects via promoting autophagy [31], reducing ROS [32] and inhibiting inflammation [33]. However, whether BA is an effective treatment for $\mathrm{SCl}$ remains unclear. Furthermore, little is known regarding BA's impact on pyroptosis. Thus, in this study, we assessed whether BA can enhance autophagy, inhibit pyroptosis, and promote functional recovery following $\mathrm{SCl}$.

\section{Materials And Methods}

\section{Animals}

Wenzhou Medical University's Experimental Animal Center (Zhejiang Province, China) supplied female C57BL/6 mice (20-30 g, license no. SCXK [ZJ] 2005-0019). All laboratory procedures involving animals followed the China National Institutes of Health's Guide for the Care and Use of Laboratory Animals, and were approved by Wenzhou Medical University's Animal Research Committee (wydw2017-0022). Best efforts were undertaken to minimize the number of animals used and pain inflicted. Standard experimental cages with a 12-h light/dark cycle were applied to house all mice individually, and unrestricted access to food and water was provided. Ninety-five mice were divided randomly into six groups: Sham ( $n=20), S C l(n=20), S C l+$ Betulinic Acid $(S C l+B A, n=20), B A+3-$ methyladenine $(B A+3 M A$, $n=20), B A+3 M A+\operatorname{MnTBAP}(n=10)$, and $B A+$ Compound $C(B A+C C, n=5)$.

\section{Reagents and Antibodies}

All reagents, antibodies and their suppliers are listed as follows: Betulinic Acid (C30H48O3, purity $\geq 98 \%)$ was obtained from MedChemExpress (Shanghai, China). MnTBAP (C96H56Cl2Mn2N8016, HPLC > 98\%, Cat. No. 475870) was obtained from Sigma-Aldrich (St. Louis, MO, USA). HE staining kit (Cat. No. G1120), 
Masson staining kit (G1340), Diaminobenzidine (DAB) developer, and pentobarbital sodium, were purchased from Solarbio Science \& Technology (Beijing, China). Dorsomorphin (Compound C, C24H25N50; purity $\geq 98.14 \%$, Cat. No. 866405-64-3) was purchased from Med Chem Express (Monmouth Junction, NJ, USA). Primary antibody against TFEB was obtained from Bethyl Laboratories (A303-673AM; Montgomery, TX, USA). Rabbit monoclonal anti-GAPDH was purchased from Biogot Technology (AP0063; Shanghai, China). The rabbit monoclonal anti-Caspase-1, anti-phosphoinositide- 3-kinase (Vps34), and anti-cathepsin D (CTSD) antibodies were purchased from Proteintech Group (12452-1, 22915-1 and 21327-1; Chicago, IL, USA). Rabbit monoclonal 3-methyladenine (3-MA) and antimicrotubule-associated 1 protein light chain 3 (LC3) antibodies were obtained from Sigma-Aldrich Chemical Co (L7543 and M9281; Milwaukee, WI, USA). NLRP3 (Cat. No. 15101), ASC (Cat. No. 67824), AMPKa (Cat. No.5832), Beclin1 (Cat. No. 3738), mTOR (Cat. No.2983), p-AMPKa (Cat. No.2535), and pmTOR (Cat. No.5536) were obtained from Cell Signaling Technology (Beverly, MA, USA). Mouse monoclonal Synaptophysin (Cat. No. ab32594), anti-SQSTM1/ p62, NeuN (Cat. No. ab177487), goat anti-rabbit IgG H\&L (Alexa Fluor® 488, Cat. No. ab150077), MAP2 (Cat. No. ab32454), and goat antimouse IgG H\&L (Alexa Fluor ${ }^{\circledR}$ 647, Cat. No. ab150115) antibodies were acquired from Abcam (Cambridge, UK). IL-1 $\beta$ (Cat. No. af5103), Antibodies against IL-18 (Cat. No. df6252), Bnip3 (Cat. No. df8188), GSDMD (Cat. No. af4013), Nix (Cat. No. df8163) and Parkin (Cat. No. af0235) were obtained from Affinity Biosciences (OH.USA). Santa Cruz Biotechnology (Dallas, TX, USA) supplied the secondary antibody Horseradish peroxidase (HRP)-conjugated immunoglobulin G (IgG). Beyotime Biotechnology (Jiangsu, China) provided the 4',6-diamidino- 2-phenylindole (DAPI), and Boyun Biotechnology (Nanjing, China) supplied the Fluorescein isothiocyanate (FITC)-conjugated IgG secondary. ThermoFisher Scientific (Rockford, IL, USA) provided the BCA kit. PerkinElmer Life Sciences (Waltham, MA, USA) supplied the Electrochemiluminescence (ECL) Plus Reagent Kit.

\section{Animal Model of $\mathrm{SCl}$ and Survival Rate Analysis}

Prior to procedure, anesthesia was administered to all animals with intraperitoneal injections of one percent $(\mathrm{w} / \mathrm{v})$ pentobarbital sodium $(50 \mathrm{mg} / \mathrm{kg})$. Then, standard laminectomy was performed at the T9T10 vertebra to expose a circle of the dura. Then, a weight-drop injury model was employed to cause a spinal contusion injury as previously described [34]. In brief, a bar with a diameter of $3.0 \mathrm{~mm}$ and a weight of $15 \mathrm{~g}$ was dropped onto the exposed spinal cord from a $15 \mathrm{~mm}$ height. After injury, muscle, fascia, and skin was closed in layers using 4-0 nonabsorbable silk sutures. Mice in the sham group $(n=20)$ received the same operation as above without weight-drop injury. Following the procedure, all mice had their bladders artificially emptied three times a day. Survival of mice was recorded on days 1, 7, 14, 21 and 28 after the procedure.

\section{Drug Administration}

All drugs were dissolved in $2 \%$ DMSO in normal saline and administered intraperitoneally. Daily injections of $20 \mathrm{mg} / \mathrm{kg}$ BA was administered to the SCl+BA group. 3-MA (15 mg/kg) and Dorsomorphin (Compound C, $1.5 \mathrm{mg} / \mathrm{kg})$ were injected $30 \mathrm{~min}$ before $B A$ administration $(20 \mathrm{mg} / \mathrm{kg})$ for $B A+3 M A$ and $B A+C C$ 
groups. For the BA + 3MA+MnTBAP group, mice received daily regimen of MnTBAP $(3 \mathrm{mg} / \mathrm{kg})$, followed by BA in 30 minutes $(20 \mathrm{mg} / \mathrm{kg})$, and 3MA 1 hour afterwards $(15 \mathrm{mg} / \mathrm{kg})$. All drugs were injected for 3 days after $\mathrm{SCl}$. All animals were sacrificed by overdose of pentobarbital sodium and histological samples were harvested on day 3, with the exception of 40 rats designated for Hematoxylin and Eosin (H\&E) and Masson staining and to assess locomotion recovery.

\section{Functional behavior assessment}

The Basso mouse scale (BMS) locomotion scale and the footprint test were administered at $0,1,7,14$, 21 , and 28 days after $\mathrm{SCl}$ to evaluate functional recovery [35]. The BMS score ranges from 0 to 9 points, with 0 indicating complete paralysis and indicating normal motor functions. Footprint analysis was performed by first immersing the hind paws in black dye, and then allowing the animals to walk for 5 min as described previously [36]. Outcome measures were measured by two independent examiners blinded to experimental conditions.

\section{Tissue slides preparation for HE and Masson staining}

On postoperative day 28 , mice ( $n=10$ per group) in the Sham, SCl, BA, and BA +3MA groups were reanesthetized with $2 \%(\mathrm{w} / \mathrm{v})$ pentobarbital sodium and perfused with normal saline, followed by addition of $4 \%(\mathrm{w} / \mathrm{v})$ paraformaldehyde in phosphate-buffered saline. Then, we separated the rostral spinal cord segments ( $1 \mathrm{~mm}$ in length, $4 \mathrm{~mm}$ far from epicenter) and the whole segments $(10 \mathrm{~mm}$ in length, epicenter in middle), then post-fixed them for $24 \mathrm{~h}$ in $4 \%(\mathrm{w} / \mathrm{v})$ paraformaldehyde. Subsequently, the each specimen was prepared for longitudinal paraffin sections after being embedded in paraffin. Using a microtome, $4 \mu \mathrm{m}$ sections were cut and mounted on poly-L-lysine-coated slides for histopathological examination by HE staining as previously described $[1,37]$. For Masson staining, we used $10 \%$ potassium dichromate and $10 \%$ trichloroacetic acid to mordant longitudinal sections and we used hematoxylin to stain nuclei. Then, using hydrochloric acid and ethanol, slides were differentiated, returned to blue with weak ammonia, and stained with the Masson solution; this staining protocol was previously described [38]. Finally, a light microscope (Olympus, Tokyo, Japan) was used to photograph images.

\section{Western blot analysis.}

After mice had been euthanized on day 3 following $\mathrm{SCl}$, the spinal cord segments in mice ( $1.5 \mathrm{~cm}$; containing the injury epicentre) ( $n=5$ per group) were dissected and stored for Western blot analyses at $-80^{\circ} \mathrm{C}$. We used protein extraction reagents to purify total proteins from spinal cord specimens and used the BCA assay to measure the proteins. $12 \%(\mathrm{w} / \mathrm{v})$ gel electrophoresis was used to separate an equal amount of $60 \mu \mathrm{g}$ protein, which were then transferred to polyvinylidene fluoride (PVDF) membranes (Roche Applied Science, Indianapolis, IN, USA). After blocking for $2 \mathrm{~h}$ at room temperature with $5 \%(\mathrm{w} / \mathrm{v})$ non-fat milk, the membranes were then incubated overnight at $4^{\circ} \mathrm{C}$ with the subsequent primary antibodies: $\operatorname{mTOR}(1: 1,000), \mathrm{p}-\mathrm{mTOR}(1: 1,000)$, AMPK $(1: 1,000), \mathrm{p}$-AMPK $(1: 1,000), \mathrm{NLRP} 3$ (1:1,000), TFEB (1:1,000), IL-1 $\beta$ (1:1,000), IL-18 (1:1,000), GSDMD (1:1,000), Nix (1:1,000), ASC $(1: 1,000)$, Parkin (1:1,000), Bnip3 (1:1000), Beclin1 (1:1,000), Caspase 1 (1:1,000), LC3 (1:500), p62 (1:1,000), CTSD 
$(1: 1,000), \operatorname{Vps} 34(1: 1,000)$, and GAPDH $(1: 1,000)$. Finally, membranes were incubated for $2 \mathrm{~h}$ at room temperature with HRP-conjugated IgG secondary antibody $(1: 5,000)$. ECL Plus Reagent Kit was used to visualize band signals, and Image Lab 3.0 software (Bio-Rad, Hercules, CA, USA) was used to quantify band intensities.

\section{Enzyme-linked immunosorbent assay (ELISA)}

We homogenized, frozen and thawed spinal cord specimens repeatedly in liquid nitrogen. Then, we centrifuged (at $10,000 \mathrm{~g}$ ) the homogenate at $4^{\circ} \mathrm{C}$ for 10 minutes, and collected the supernatant for ELISA. ELISA kits were used to evaluate the levels of AOPP, 8-OHdG, and MDA in spinal cord specimens according to manufacturer's protocols (Boyun Biotech, Shanghai, China). Finally, the quantifications of AOPP, 8-OHdG, and MDA were performed using a microplate reader at $550 \mathrm{~nm}$ with a correction wavelength of $450 \mathrm{~nm}$.

\section{Immunofluorescence Staining}

Immunofluorescence staining for tissue sections were performed as previously described [32]. We deparaffinized, rehydrated, washed, and then treated sections at $95^{\circ} \mathrm{C}$ for 20 min with $10.2 \mathrm{mM}$ sodium citrate buffer. Then, $0.1 \%(\mathrm{v} / \mathrm{v})$ PBS-Triton X-100 was used to permeabilize the sections (10 minutes), and used 10\% (v/v) bovine serum albumin in PBS was used for blocking (1 hour). After that, slides were incubated overnight at $4^{\circ} \mathrm{C}$ with antibodies against p62 (1:200)/NeuN (1:400), LC3 (1:200)/NeuN (1:400), GSDMD (1:150)/NeuN (1:400), Caspase-1/NeuN, and SYN (1:200)/NeuN (1:400), Nix (1:150)/NeuN (1:400), and MAP2 (1:200). Then, we washed the sections for 10 minutes at room temperature three times and incubated the sections at room temperature for 1 hour with FITC-conjugated secondary antibody. Finally, we captured and assessed images taken by a fluorescence microscope (Olympus, Tokyo, Japan) in 6 randomly selected fields from 3 random sections of each sample.

\section{Statistical Analyses}

All statistical analyses were performed on SPSS 19.0 (SPSS, Chicago, IL). All data are presented as mean \pm Standard Error of Mean (SEM). Independent-sample t-test and one-way ANOVA with LSD (equal variances assumed) were used as appropriate. $P<0.05$ was considered statistically significant.

\section{Results}

\subsection{BA promotes functional recovery.}

Compared to sham control, the $\mathrm{SCl}$ group showed an expanded area of glial scar $(p<0.001)$, downregulated MAP2 $(p<0.001)$, and less SYN-positive synapses onto ventral motor neurons $(p<0.001)$. With BA treatment, animals had less glial scar, higher neuronal MAP2 expression, and more SYN-positive synapses onto ventral motor neurons compared with the untreated SCI group $(p<0.001, p<0.001, p=$ 0.006 , respectively; Fig. 1A-F). Moreover, footprint analysis showed that the BA group fared better than $\mathrm{SCl}$ group for functional recovery at 28 days after injury (Fig. 1G). For the Sham group, the BMS scores 
were notably higher compared to the $\mathrm{SCl}$ group at days $1,7,14,21$ and 28 post-procedure $(\mathrm{p}<0.001$ for all). Similarly, the BA group also had higher BMS scores at days 1, 7, 14, 21 and 28 post-procedure compared with the $\mathrm{SCl}$ group $(P=0.018, P=0.021, P=0.022, P=0.021, P=0.005$, respectively; Fig. $1 \mathrm{H})$. Finally, analysis of survival rate revealed that the BA group fared better than the $\mathrm{SCl}$ group $(P=0.027$; Fig. 1I). Together, these results demonstrate that BA promotes functional recovery and survival following SCl.

\subsection{BA attenuates pyroptosis after SCl.}

ASC, GSDMD, Caspase-1, NLRP3, IL-1 3 , and IL-18 were assessed in the spinal cord after SCl to assess pyroptotic activity in Sham, SCl and BA groups. As shown in Fig. 2A-D, immunofluorescence staining showed that Caspase-1 and GSDMD density in neurons were significantly increased in the spinal cord lesions in the $\mathrm{SCl}$ group relative to the Sham group $(P<0.001$ for both), while BA decreased the densities of Caspase-1 and GSDMD compared with the SCI group ( $p=0.016, p=0.013$, respectively). Western blots for ASC, GSDMD, Caspase-1, NLRP3, IL-1 3 , and IL-18 expressions levels were also assessed (Fig. 2E). Results demonstrated that the OD values for ASC, Caspase-1, GSDMD, IL-1 $\beta$, IL-18 and NLRP3 were higher in the $\mathrm{SCl}$ group compared with the Sham group $(P<0.001$ for all), and that BA decreases in the OD values for these markers relative to the $\mathrm{SCl}$ group $(P<0.001, P=0.009, P=0.003, P=0.004, P=$ $0.004, P=0.013$, respectively; Fig. $2 \mathrm{~F}$ ). These results suggest that BA reduces pyroptosis-related markers, suggestive of an inhibitory effect on pyroptosis following SCl.

\subsection{BA enhances autophagy after SCl.}

To assess autophagic activity in the spinal cord lesion after $\mathrm{SCl}$, we measured protein levels of autophagosomal markers (LC3II, Beclin1 and Vps34), an autolysosome-related marker (CTSD), and an autophagic substrate protein (p62). As shown in Fig. 3A, immunofluorescence staining revealed p62 levels in the neurons at the lesion; a green label was used to mark p62 was labeled green, a red label for neurons, and a blue label for nuclei. Quantitative analyses showed that after $\mathrm{SCl}$, the percentage of p62positive neurons significantly increased $(P<0.001)$; however, the BA group had a lower percentage of p62-positive neurons compared with the SCI group ( $P<0.001$; Fig. 3B). To assess LC3II levels, a green label was applied for LC3II, a red label for neurons (NeuN), and blue label for nuclei (DAPI). As shown in Fig. 3C, the spinal cord exhibited higher LC3 $\triangle$ signals in the SCl group than in the Sham group $(P<0.001)$; BA treatment further increased LC3 $₫$ signals compared with the $\mathrm{SCl}$ group $(P<0.001$; Fig. 3D). The amount of p62, LC3II, Beclin1, Vps34, and CTSD proteins were measured by Western blot (Fig. 3E). Results showed that the OD of p62, LC3II, Beclin1, Vps34, were higher in the SCI group than in the Sham group ( $P<0.001, P=0.001, P=0.001, P<0.001$, respectively), with lower OD values for CTSD in the SCI group $(P<0.001)$. BA enhanced the level of LC3II, Beclin1, Vps34, and CTSD as well as decreased level of p62 in the BA group compared with the $\mathrm{SCl}$ group ( $\mathrm{p}<0.001$ for all; Fig. 3F). These results recapitulate the known phenomenon that following $\mathrm{SCl}$, autophagy substrates accumulate despite an upregulation autophagosome- and autolysosome-related markers. These results also demonstrate that BA is not only able to increase autophagosome- and autolysosome-related markers, it also alleviates autophagy substrate burden, likely due to an inducing an overall increase in autophagic activity following $\mathrm{SCl}$. 


\subsection{Inhibition of autophagy reverses the effects of BA on pyroptosis after SCl.}

3MA, an autophagy inhibitor, was co-administered with BA to assess whether BA's beneficial effects on outcomes following $\mathrm{SCl}$ is due to autophagy activation. Immunofluorescence and neuron co-localization analyses revealed increased $p 62$ density and decreased LC3区 signals BA+3MA group compared with the BA group $(P<0.001, P<0.001$, respectively; Fig. 4A-D). The expression levels of p62, LC3II, Beclin1, Vps34, and CTSD were detected by Western blot (Fig. 4E). Results showed that the OD values for LC3II, Beclin1, Vps34, and CTSD were lower in the BA+3MA group than in the BA group $(P=0.004, P=0.001, P$ $=0.017, P<0.001$, respectively), with a higher OD value for p62 in the BA+3MA group $(P=0.001$; Fig. 4F). These results demonstrate that $3 \mathrm{MA}$ was effective in inhibiting autophagy when co-administered with BA. Next, pyroptotic activity was assessed in BA-treated animals via immunofluorescence staining and Western blotting after co-administration of 3-MA. Immunofluorescence showed that Caspase-1 and GSDMD densities in neurons were higher in the BA+3MA group than in the BA group $(P<0.001$ for both; Fig. 4G-J). The expression levels of ASC, GSDMD, Caspase-1, NLRP3, IL-1 $\beta$, and IL-18 were also measured by Western blot (Fig. 4K). Results revealed that the OD values for ASC, Caspase-1, GSDMD, IL-1 3 , IL-18 and NLRP3 were higher in the BA+3MA group than in the BA group $(P<0.001, P<0.001, P=0.001, P$ $=0.002, P=0.002, P<0.001$ respectively; Fig. $4 \mathrm{~L}$ ). These results show that co-administration of 3MA with BA leads to a reduction in BA's effect on reducing pyroptosis, suggesting that the autophagy-enhancing effects of BA may underly the mechanism by which it inhibits pyroptosis.

\subsection{Autophagy inhibition reverts the effects of BA on functional recovery after SCl.}

Compared to the BA group, the BA+3MA group showed an increased area of glial scar $(P=0.005$, Fig. 5AB), decreased MAP2 levels $(P<0.001$, Fig. 5C-D) and lower number of SYN-positive synapses onto ventral motor neurons following SCI $(P<0.001$, Fig. 5E-F). At day 28 after injury, the BA group showed a significant restoration of hind legs movement with coordinated crawling, whereas the BA+3MA group was still dragging their hind legs (Fig. 5G). In the BA+3MA group, the BMS scores were significantly lower than those in the BA group after $\mathrm{SCl}$ at days $1,7,14,21$ and $28(P=0.032, P=0.017, P=0.025, P=0.008, P=$ 0.005 , respectively; Fig. $5 \mathrm{H}$ ). Furthermore, the survival rate of the BA+3MA group was notably lower than the BA group ( $P=0.033$, Fig. $5 \mathrm{l})$. These results suggest that BA's autophagy enhancing effects may be responsible for improved outcomes and survival with $\mathrm{BA}$ treatment following $\mathrm{SCl}$.

\subsection{BA enhances mitophagy and reduces ROS accumulation after SCl.}

ROS oxidation products - AOPP, 8-OHdG, and MDA - were measured by ELISA to evaluate changes in ROS levels after $\mathrm{SCl}$. The levels of AOPP, 8-OHdG, and MDA were all higher in the $\mathrm{SCl}$ group compared with the Sham group ( $P<0.001$ for all). BA treatment decreased the levels of AOPP, 8-OHdG, and MDA ( $P$ $<0.001, P<0.001, P=0.041$, respectively), and the levels of AOPP, 8-OHdG, and MDA were all higher in the $\mathrm{BA}+3 \mathrm{MA}$ group compared with the BA group $(P=0.004, P=0.010, P=0.013$, respectively, Fig. 6A). Biomarkers Bnip3, Nix and Parkin assessed to measure mitophagy in the Sham, SCl, BA and BA+3MA groups. Immunofluorescence showed that there were significantly more Nix-positive neurons in the SCI group relative to the Sham group $(P=0.026)$, and more Nix-positive neurons were observed in the BA 
group compared with the $\mathrm{SCl}$ group $(P=0.001$; Fig. 6B, C). Furthermore, compared to BA group, there were more Nix-positive neurons in the BA+3MA group ( $P<0.001$; Fig. 6D, E). The expression levels of Bnip3, Nix and Parkin were also measured by Western blot (Fig. 6F, H). Results showed that Bnip3, Nix and Parkin levels were significantly higher in the SCI group compared to the Sham group $(P=0.001, P=$ $0.002, P=0.001$, respectively), and these levels were even higher in the BA group compared with the SCI group ( $P=0.001, P<0.001, P=0.003$, respectively; Fig. 6 G). Finally, compared to BA group, the expression levels of Bnip3, Nix and Parkin in the BA+3MA group were all higher $(P=0.041, P=0.018, P=$ 0.002, respectively; Fig. 6I). Together, these results indicate that BA reduces ROS accumulation and augments mitophagy in $\mathrm{SCl}$, and that these effects are likely due to its autophagy enhancing effects.

\section{BA inhibits pyroptosis via mitophagy-induced ROS elimination.}

We tested whether BA inhibits pyroptosis via mitophagy-induced ROS elimination in neurons after SCl. MnTBAP, a ROS scavenger, was administered prior to treatment with BA and 3MA. Results showed that MnTBAP decreased AOPP, 8-OHdG, and MDA levels in BA+3MA treated mice $(P=0.001, P=0.001, P=$ 0.003, respectively; Fig. 7A). Biomarkers ASC, GSDMD, Caspase-1, NLRP3, IL-1 $\beta$, and IL-18 were evaluated to assess pyroptosis in the BA+3MA and BA+3MA+MnTBAP groups. As shown in Fig. 7B-E,

immunofluorescence staining showed that Caspase-1 and GSDMD densities in neurons were significantly decreased in the spinal cord lesions in the BA+3MA+MnTBAP group relative to the BA+3MA group $(P<$ 0.001 for both). Western blots for ASC, Caspase-1, GSDMD, IL-1 $\beta$, IL-18 and NLRP3 expressions levels were assessed for each group (Fig. 7F), and results showed that the OD values for ASC, Caspase-1, GSDMD, IL-1 $\beta$, IL-18 and NLRP3 were lower in the BA+3MA+MnTBAP group compared with the BA+3MA group $(P=0.029, P<0.001, P<0.001, P<0.001, P=0.002, P<0.001$, respectively; Fig. $7 \mathrm{G})$. These results suggest that the pyroptosis inhibiting effects of BA may be due to its mitophagy-activating and ROSreducing effects.

\subsection{BA activates autophagy and mitophagy via enhancing AMPK-mTOR -TFEB activity.}

Finally, we sought to investigate the mechanism underlying how BA modulates pyroptosis, autophagy and mitophagy. We assessed whether TFEB, a known activator of autophagy, is involved. Western blot analysis revealed that $\mathrm{p}-\mathrm{AMPK}$ and TFEB levels were higher in the $\mathrm{SCl}$ group compared with the Sham group $(P=0.038, P=0.008$, respectively), while $\mathrm{p}$-mTOR was lower $(P=0.005)$. BA increased the levels of p-AMPK and TFEB and decreased p-mTOR level $(P=0.039, P=0.044, P=0.004$, respectively, Fig. 8A, D). Next, we explored the effects of compound $C$ (CC), an AMPK blocker, on the effects of BA. Here, p-AMPK and TFEB levels were lower in the BA+CC group compared with the BA group $(P<0.001, P=0.001$, respectively), while $p-m T O R$ levels in the $B A+C C$ group was higher $(P=0.008, \mathbf{F i g} . \mathbf{8 B}, E)$. We also evaluated whether the AMPK-mTOR -TFEB axis is also involved in the mechanism by which BA modulates pyroptosis, autophagy, and mitophagy related proteins. Our results demonstrated that Caspase-1, NLRP3 and GSDMD levels were higher in the BA+CC group when compared to the BA group ( $P$ $=0.046, P=0.018, P=0.012$, respectively); 62 levels were higher in the $\mathrm{BA}+\mathrm{CC}$ group when compared to the BA group $(P=0.004)$, with a lower level of LC3II $(P=0.009)$. Finally, Bnip3, Nix and Parkin were lower 
in the BA+CC group when compared to the BA group $(P=0.012, P=0.005, P<0.001$, respectively, Fig. 8C, F). Together, these findings suggest that BA activates autophagy, inhibits pyroptosis, and augments mitophagy via the AMPK-mTOR-TFEB pathway.

\section{Discussion}

Betulinic acid (BA), a natural pentacyclic triterpenoid, has gained considerable attention in recent years for its strong biological and medicinal properties [39]. Increasing evidence suggest that BA plays a substantial role in the treatment of various nervous system diseases such Alzheimer's disease [40] peripheral neuropathies [41]. Spinal cord injury is a devastating and common disease that inflicts substantial physiological, emotional, and economic damage to patients, their families and societies worldwide. In this study, we present novel preclinical evidence that BA may contribute functional and survival outcomes following SCl. Mechanistically, our results demonstrate that BA's therapeutic effect was likely due to autophagy augmentation via the AMPK-mTOR-TFEB pathway, subsequently inducing mitophagy, suppressing ROS accumulation, and inhibiting pyroptosis.

Autophagy, a lysosomal-dependent degradation pathway for intracellular proteins and organelles, plays an important role in human health and disease [42]. In the context of $\mathrm{SCl}$, autophagy has shown to play an important role, albeit a complicated one. On one hand, melatonin-induced increase in autophagy has been shown to promote locomotor recovery in SCI [43], but on the other hand, induction of autophagy may lead to neuronal cell death [44]. Despite lingering controversy, accumulating evidence seems to suggest that autophagy is primarily beneficial in the context of $\mathrm{SCl}[45,46]$. Our previous work revealed that BA was an effective activator of autophagy [47], and thus, we hypothesized that BA may be effective in improving outcomes following SCl. In the present study, not only did we find that BA improved outcomes following $\mathrm{SCl}$, WB and immunofluorescence staining results also suggested that these beneficial effects are largely due to up-regulation of autophagy, and that 3MA reverses these benefits.

Pyroptosis is another cellular pathway that has also been implicated in a variety of pathologies such as colitis, myocardial dysfunction, and neuronal damage [48-50]. In brief, pyroptosis is a form of inflammatory programmed cell death characterized by inflammasome activation, membrane poreformation, swelling, rupture, and ultimate dissolution and release of intracellular contents [51]. In the canonical inflammasome pathway, NLRP3 inflammasomes are activated to recruit ASC to form ASC focus $[51,52]$. Then, ASC focus activated Caspase- 1 , which cleaves pro-IL-18/1 $\beta$ and gasdermin D (GSDMD) to release their mature forms [51]. IL-18/1 $\beta$ and GSDMD are then released to the extracellular domain and subsequently trigger a robust inflammatory response [53]. In this study, we hypothesized that BA's augmentation of autophagy may be able to suppress pyroptosis in SCl. Our Western blot and immunofluorescence results showed that BA was able to significantly depressed pyroptosis-associated markers such as ASC, NLRP3, GSDMD, Caspase-1, IL-1 $\beta$ and IL-1B, suggesting that BA may be an effective inhibitor of pyroptosis in SCl. Furthermore, we found that 3-MA co-administration abates these pyroptosis inhibiting effects, suggesting that BA likely suppresses pyroptosis via enhancing autophagy. 
Increasing evidence demonstrates that the secondary injury stage after $\mathrm{SCl}$ is closely related to mitochondrial injury and excessive ROS generation [54]. Damaged mitochondria leads to a massive accumulation of ROS, which can induce NLRP3 inflammasome activation and subsequently trigger caspase-1-dependent pyroptosis $[55,56]$. Mitophagy, a selective autophagic degradation of damaged mitochondria [57], can reduce ROS accumulation [58, 59], and may play a central role in curtailing pyroptosis following SCl. To explore this postulation, we investigated whether BA-mediated autophagy enhancement promotes mitophagy, and whether mitophagy underlies the mechanism by which BA inhibits pyroptosis following SCl. Our results showed that BA significantly elevated the levels of Bnip3, Nix and Parkin following $\mathrm{SCl}$, suggesting an upregulation of mitophagy. Furthermore, our findings demonstrate that BA decreased ROS oxidation products, including 8-OHdG, AOPP and MDA. These changes were abated by co-administration of 3-MA, suggesting that mitophagy activation and ROS reduction occurred downstream of autophagy enhancement. We also found that MnTBAP, a ROS scavenger, decreased expressions levels of the pyroptosis group, suggesting that in $\mathrm{SCl}$, ROS is likely responsible for pyroptosis activation, and that autophagy-induced mitophagy activation and ROS reduction may underly the mechanism by which BA suppresses pyroptosis.

In order to further to elucidate the underlying mechanism of how BA promotes autophagy in $\mathrm{SCl}$, we also examined upstream mechanisms of autophagy. Transcription factor EB (TFEB) is a central regulator of autophagy $[60,61]$. Past studies have shown that TFEB activation occurs in response to a variety of cellular stress [62], which impacts the ATP to ADP ratio, triggering the phosphorylation of AMPK. Subsequently, AMPK regulates cell metabolism [63], inhibiting mTOR (a master regulator of cell growth and metabolism) in the cytoplasm to allow nuclear translocation of TFEB [64]. In our present work, we showed that BA increases the expression of TFEB. Furthermore, our results revealed that BA leads to increased phosphorylation of AMPK and inhibited the phosphorylation of mTOR. Finally, using compound C (CC), an AMPK blocker, we showed that inhibition of AMPK-mTOR-TFEB signaling pathway led to diminished effects of BA on autophagy, and mitophagy, and pyroptosis.

Naturally, there are several limitations in our study that need to be further investigated. For example, previous studies revealed that AMPK-SPK2-CARM1 signaling pathway is another important pathway regulating TFEB in the nucleus $[65,66]$, and future investigations should explore whether BA also acts through AMPK -SPK2-CARM1-TFEB signaling pathway in SCl. Studies have shown that besides ROS, CTSB and $\mathrm{K}+$ can also cause pyroptosis $[67,68]$, and future studies should also explore whether BA influences $\mathrm{CTSB}$ and $\mathrm{K}+$ in SCl. Finally, while these preclinical findings are promising, more work is needed to be done to explore optimal dosing and toxicities of $\mathrm{BA}$ in the treatment of $\mathrm{SCl}$ prior to clinical translation.

\section{Conclusions}

Our findings demonstrate that BA activates the AMPK-mTOR-TFEB signaling pathway, which enhances autophagy in SCl. Increased autophagy induces mitophagy and reduces ROS accumulation, subsequently inhibiting pyroptosis. Ultimately, these effects of BA culminate in improved outcomes and 
survival following $\mathrm{SCl}$. A schematic illustration of our findings is presented in Fig. 8G. Overall, these results provide novel preclinical evidence demonstrating the therapeutic benefit of $\mathrm{BA}$ in SCl. Future investigations are now eagerly awaited to further the clinical translation of $\mathrm{BA}$ as a treatment for $\mathrm{SCl}$ patients.

\section{Abbreviations}

SCl: Spinal cord injury; BA: Betulinic acid; CNS: Central nervous system; ROS: Reactive oxygen species; BMS: Basso mouse scale; GSDMD: Gasdermin D; TFEB: Transcription factor; CC: Compound C;

\section{Declarations}

\section{Acknowledgments}

Not applicable

\section{Authors' contributions}

Kailiang Zhou, Wenfei Ni and Hui Xu designed and supervised this study. Chenyu Wu and Huanwen Chen conducted the majority of the experiments and completed the manuscript. Rong Zhuang, Yongli Wang, Xinli Hu and Haojie Zhang analyzed the data. Chenyu Wu, Yu Xu and Jiafeng Li participated the experiments and the manuscript writing. Yao Li and Huazi Xu participated in editing the manuscript. Hui $\mathrm{Xu}$ and Wenfei Ni the spinal cord injury model. All authors approved the final version of the manuscript.

\section{Funding}

This study was supported by Grants from Zhejiang Provincial Public Welfare Technology Application Research Project (Grant no. LGF20H150003 to Kailiang Zhou), Zhejiang Provincial Medicine and Health Technology Project (Grant no. 2017KY472 to Kailiang Zhou), the Natural Science Foundation of China (Grant no. 82072192 and Grant no.81601705 to Kailiang Zhou, Grant no. 81873992 to Huazi Xu).

\section{Ethics Approval and consent to participate}

All animal procedures were performed under the guidelines of the institutional review board and the ethics committee of Wenzhou Medical University.

\section{Consent for publication}

Not applicable

\section{Declaration of competing interest}

The authors declare no conflicts of interest. 


\section{References}

[1] K.L. Zhou, Y.F. Zhou, K. Wu, N.F. Tian, Y.S. Wu, Y.L. Wang, D.H. Chen, B. Zhou, X.Y. Wang, H.Z. Xu, X.L. Zhang, Stimulation of autophagy promotes functional recovery in diabetic rats with spinal cord injury, Sci Rep 5 (2015) 17130.

[2] S. Liu, C. Sarkar, M. Dinizo, A.I. Faden, E.Y. Koh, M.M. Lipinski, J. Wu, Disrupted autophagy after spinal cord injury is associated with ER stress and neuronal cell death, Cell Death Dis 6(1) (2015) e1582.

[3] M.B. Bracken, M.J. Shepard, W.F. Collins, T.R. Holford, W. Young, D.S. Baskin, H.M. Eisenberg, E. Flamm, L. Leo-Summers, J. Maroon, et al., A randomized, controlled trial of methylprednisolone or naloxone in the treatment of acute spinal-cord injury. Results of the Second National Acute Spinal Cord Injury Study, N Engl J Med 322(20) (1990) 1405-11.

[4] D. Xu, L. Yang, Y. Li, Y. Sun, Clinical study of ganglioside (GM) combined with methylprednisolone (MP) for early acute spinal injury, Pakistan journal of pharmaceutical sciences 28(2 Suppl) (2015) 701-4.

[5] M.G. Fehlings, D.H. Nguyen, Immunoglobulin G: a potential treatment to attenuate neuroinflammation following spinal cord injury, Journal of clinical immunology 30 Suppl 1(Suppl 1) (2010) S109-12.

[6] H.Y. Zhang, X. Zhang, Z.G. Wang, H.X. Shi, F.Z. Wu, B.B. Lin, X.L. Xu, X.J. Wang, X.B. Fu, Z.Y. Li, C.J. Shen, X.K. Li, J. Xiao, Exogenous basic fibroblast growth factor inhibits ER stress-induced apoptosis and improves recovery from spinal cord injury, CNS neuroscience \& therapeutics 19(1) (2013) 20-9.

[7] C. Penas, M.S. Guzmán, E. Verdú, J. Forés, X. Navarro, C. Casas, Spinal cord injury induces endoplasmic reticulum stress with different cell-type dependent response, Journal of neurochemistry 102(4) (2007) 1242-55.

[8] K.V. Ambrozaitis, E. Kontautas, B. Spakauskas, D. Vaitkaitis, [Pathophysiology of acute spinal cord injury], Medicina (Kaunas, Lithuania) 42(3) (2006) 255-61.

[9] X.B. Chen, Z.L. Wang, Q.Y. Yang, F.Y. Zhao, X.L. Qin, X.E. Tang, J.L. Du, Z.H. Chen, K. Zhang, F.J. Huang, Diosgenin Glucoside Protects against Spinal Cord Injury by Regulating Autophagy and Alleviating Apoptosis, Int J Mol Sci 19(8) (2018).

[10] W. Dai, X. Wang, H. Teng, C. Li, B. Wang, J. Wang, Celastrol inhibits microglial pyroptosis and attenuates inflammatory reaction in acute spinal cord injury rats, Int Immunopharmacol 66 (2019) 215223.

[11] M. He, Y. Ding, C. Chu, J. Tang, Q. Xiao, Z.G. Luo, Autophagy induction stabilizes microtubules and promotes axon regeneration after spinal cord injury, Proceedings of the National Academy of Sciences of the United States of America 113(40) (2016) 11324-11329. 
[12] H.Q. Duan, Q.L. Wu, X. Yao, B.Y. Fan, H.Y. Shi, C.X. Zhao, Y. Zhang, B. Li, C. Sun, X.H. Kong, X.F. Zhou, S.Q. Feng, Nafamostat mesilate attenuates inflammation and apoptosis and promotes locomotor recovery after spinal cord injury, 24(5) (2018) 429-438.

[13] J.P. de Rivero Vaccari, G. Lotocki, A.E. Marcillo, W.D. Dietrich, R.W. Keane, A molecular platform in neurons regulates inflammation after spinal cord injury, The Journal of neuroscience : the official journal of the Society for Neuroscience 28(13) (2008) 3404-14.

[14] J.P. de Rivero Vaccari, W.D. Dietrich, R.W. Keane, Activation and regulation of cellular inflammasomes: gaps in our knowledge for central nervous system injury, Journal of cerebral blood flow and metabolism : official journal of the International Society of Cerebral Blood Flow and Metabolism 34(3) (2014) 369-75.

[15] G. Trendelenburg, Molecular regulation of cell fate in cerebral ischemia: role of the inflammasome and connected pathways, Journal of cerebral blood flow and metabolism : official journal of the International Society of Cerebral Blood Flow and Metabolism 34(12) (2014) 1857-67.

[16] Q. Fu, J. Wu, X.Y. Zhou, M.H. Ji, Q.H. Mao, Q. Li, M.M. Zong, Z.Q. Zhou, J.J. Yang, NLRP3/Caspase-1 Pathway-Induced Pyroptosis Mediated Cognitive Deficits in a Mouse Model of Sepsis-Associated Encephalopathy, Inflammation 42(1) (2019) 306-318.

[17] F. Marín-Aguilar, J. Ruiz-Cabello, M.D. Cordero, Aging and the Inflammasomes, Experientia supplementum (2012) 108 (2018) 303-320.

[18] W.P. Lin, G.P. Xiong, Q. Lin, X.W. Chen, L.Q. Zhang, J.X. Shi, Q.F. Ke, J.H. Lin, Heme oxygenase-1 promotes neuron survival through down-regulation of neuronal NLRP1 expression after spinal cord injury, J Neuroinflammation 13(1) (2016) 52.

[19] Z. Qiu, Y. He, H. Ming, S. Lei, Y. Leng, Z. Xia, Lipopolysaccharide (LPS) Aggravates High Glucose- and Hypoxia/Reoxygenation-Induced Injury through Activating ROS-Dependent NLRP3 InflammasomeMediated Pyroptosis in H9C2 Cardiomyocytes, Journal of diabetes research 2019 (2019) 8151836.

[20] P.G. Sullivan, S. Krishnamurthy, S.P. Patel, J.D. Pandya, A.G. Rabchevsky, Temporal characterization of mitochondrial bioenergetics after spinal cord injury, Journal of neurotrauma 24(6) (2007) 991-9.

[21] D. Gozuacik, A. Kimchi, Autophagy as a cell death and tumor suppressor mechanism, Oncogene 23(16) (2004) 2891-906.

[22] D. Glick, S. Barth, K.F. Macleod, Autophagy: cellular and molecular mechanisms, The Journal of pathology 221(1) (2010) 3-12.

[23] X. Zhang, H. Yan, Y. Yuan, J. Gao, Z. Shen, Y. Cheng, Y. Shen, R.R. Wang, X. Wang, W.W. Hu, G. Wang, Z. Chen, Cerebral ischemia-reperfusion-induced autophagy protects against neuronal injury by mitochondrial clearance, Autophagy 9(9) (2013) 1321-33. 
[24] C. Wu, H. Xu, J. Li, X. Hu, X. Wang, Y. Huang, Y. Li, S. Sheng, Y. Wang, H. Xu, W. Ni, K. Zhou, Baicalein Attenuates Pyroptosis and Endoplasmic Reticulum Stress Following Spinal Cord Ischemia-Reperfusion Injury via Autophagy Enhancement, Front Pharmacol 11 (2020) 1076.

[25] J. Hu, H. Han, P. Cao, W. Yu, C. Yang, Y. Gao, W. Yuan, Resveratrol improves neuron protection and functional recovery through enhancement of autophagy after spinal cord injury in mice, Am J Transl Res 9(10) (2017) 4607-4616.

[26] I. Kim, S. Rodriguez-Enriquez, J.J. Lemasters, Selective degradation of mitochondria by mitophagy, Archives of biochemistry and biophysics 462(2) (2007) 245-53.

[27] B.G. Byrne, J.F. Dubuisson, A.D. Joshi, J.J. Persson, M.S. Swanson, Inflammasome components coordinate autophagy and pyroptosis as macrophage responses to infection, mBio 4(1) (2013) e0062012.

[28] W. Liu, S. Li, Z. Qu, Y. Luo, R. Chen, S. Wei, X. Yang, Q. Wang, Betulinic acid induces autophagymediated apoptosis through suppression of the PI3K/AKT/mTOR signaling pathway and inhibits hepatocellular carcinoma, Am J Transl Res 11(11) (2019) 6952-6964.

[29] P. Yogeeswari, D. Sriram, Betulinic acid and its derivatives: a review on their biological properties, Current medicinal chemistry 12(6) (2005) 657-66.

[30] M.S. Planchard, M.A. Samel, A. Kumar, V. Rangachari, The natural product betulinic acid rapidly promotes amyloid- $\beta$ fibril formation at the expense of soluble oligomers, ACS chemical neuroscience 3(11) (2012) 900-8.

[31] S. Giordano, V. Darley-Usmar, J. Zhang, Autophagy as an essential cellular antioxidant pathway in neurodegenerative disease, Redox Biol 2 (2014) 82-90.

[32] J. Li, Q. Wang, H. Cai, Z. He, H. Wang, J. Chen, Z. Zheng, J. Yin, Z. Liao, H. Xu, J. Xiao, F. Gong, FGF1 improves functional recovery through inducing PRDX1 to regulate autophagy and anti-ROS after spinal cord injury, Journal of cellular and molecular medicine 22(5) (2018) 2727-2738.

[33] Y. Chen, J. Meng, Q. Xu, T. Long, F. Bi, C. Chang, W. Liu, Rapamycin improves the neuroprotection effect of inhibition of NLRP3 inflammasome activation after TBI, Brain Res 1710 (2019) 163-172.

[34] K.R. Byrnes, B.A. Stoica, S. Fricke, S. Di Giovanni, A.I. Faden, Cell cycle activation contributes to postmitotic cell death and secondary damage after spinal cord injury, Brain 130(Pt 11) (2007) 2977-92.

[35] Z. He, S. Zou, J. Yin, Z. Gao, Y. Liu, Y. Wu, H. He, Y. Zhou, Q. Wang, J. Li, F. Wu, H. Xu, X. Jia, J. Xiao, Inhibition of Endoplasmic Reticulum Stress Preserves the Integrity of Blood-Spinal Cord Barrier in Diabetic Rats Subjected to Spinal Cord Injury, Scientific reports 7(1) (2017) 7661. 
[36] J. Lee, H. Choi, H. Ahn, B. Ju, T. Yune, Matrix metalloproteinase-3 promotes early blood-spinal cord barrier disruption and hemorrhage and impairs long-term neurological recovery after spinal cord injury, The American journal of pathology 184(11) (2014) 2985-3000.

[37] K. Wu, K. Zhou, Y. Wang, Y. Zhou, N. Tian, Y. Wu, D. Chen, D. Zhang, X. Wang, H. Xu, X. Zhang, Stabilization of HIF-1a by FG-4592 promotes functional recovery and neural protection in experimental spinal cord injury, Brain Res 1632 (2016) 19-26.

[38] H. Li, C. Wang, T. He, T. Zhao, Y.Y. Chen, Y.L. Shen, X. Zhang, L.L. Wang, Mitochondrial Transfer from Bone Marrow Mesenchymal Stem Cells to Motor Neurons in Spinal Cord Injury Rats via Gap Junction, Theranostics 9(7) (2019) 2017-2035.

[39] A. Saneja, D. Arora, R. Kumar, R.D. Dubey, A.K. Panda, P.N. Gupta, Therapeutic applications of betulinic acid nanoformulations, Annals of the New York Academy of Sciences 1421(1) (2018) 5-18.

[40] S.P. Navabi, A. Sarkaki, E. Mansouri, M. Badavi, A. Ghadiri, Y. Farbood, The effects of betulinic acid on neurobehavioral activity, electrophysiology and histological changes in an animal model of the Alzheimer's disease, Behavioural brain research 337 (2018) 99-106.

[41] S.S. Bellampalli, Y. Ji, A. Moutal, S. Cai, E.M.K. Wijeratne, M.A. Gandini, J. Yu, A. Chefdeville, A. Dorame, L.A. Chew, C.L. Madura, S. Luo, G. Molnar, M. Khanna, J.M. Streicher, G.W. Zamponi, A.A.L. Gunatilaka, R. Khanna, Betulinic acid, derived from the desert lavender Hyptis emoryi, attenuates paclitaxel-, HIV-, and nerve injury-associated peripheral sensory neuropathy via block of $\mathrm{N}$ - and T-type calcium channels, Pain 160(1) (2019) 117-135.

[42] N. Mizushima, B. Levine, A. Cuervo, D. Klionsky, Autophagy fights disease through cellular selfdigestion, Nature 451(7182) (2008) 1069-75.

[43] Y. Li, Y. Guo, Y. Fan, H. Tian, K. Li, X. Mei, Melatonin Enhances Autophagy and Reduces Apoptosis to Promote Locomotor Recovery in Spinal Cord Injury via the PI3K/AKT/mTOR Signaling Pathway, Neurochemical research 44(8) (2019) 2007-2019.

[44] Y. Uchiyama, M. Koike, M. Shibata, Autophagic neuron death in neonatal brain ischemia/hypoxia, Autophagy 4(4) (2008) 404-8.

[45] Z. Li, F. Liu, L. Zhang, Y. Cao, Y. Shao, X. Wang, X. Jiang, Z. Chen, Neuroserpin restores autophagy and promotes functional recovery after acute spinal cord injury in rats, Molecular medicine reports $17(2)$ (2018) 2957-2963.

[46] P. Tang, H. Hou, L. Zhang, X. Lan, Z. Mao, D. Liu, C. He, H. Du, L. Zhang, Autophagy reduces neuronal damage and promotes locomotor recovery via inhibition of apoptosis after spinal cord injury in rats, Molecular neurobiology 49(1) (2014) 276-87. 
[47] J. Li, G. Bao, A.L. E, J. Ding, S. Li, S. Sheng, Z. Shen, Z. Jia, C. Lin, C. Zhang, Z. Lou, H. Xu, W. Gao, K. Zhou, Betulinic Acid Enhances the Viability of Random-Pattern Skin Flaps by Activating Autophagy, Front Pharmacol 10 (2019) 1017.

[48] C. Ma, D. Yang, B. Wang, C. Wu, Y. Wu, S. Li, X. Liu, K. Lassen, L. Dai, S. Yang, Gasdermin D in macrophages restrains colitis by controlling cGAS-mediated inflammation, Science advances $6(21)$ (2020) eaaz6717.

[49] Y. Liang, P. Song, Y. Zhu, J. Xu, P. Zhu, R. Liu, Y. Zhang, TREM-1-targeting LP17 attenuates cerebral ischemia-induced neuronal injury by inhibiting oxidative stress and pyroptosis, Biochemical and biophysical research communications 529(3) (2020) 554-561.

[50] F. He, G. Zheng, J. Hou, Q. Hu, Q. Ling, G. Wu, H. Zhao, J. Yang, Y. Wang, L. Jiang, W. Tang, Z. Yang, Nacetylcysteine alleviates post-resuscitation myocardial dysfunction and improves survival outcomes via partly inhibiting NLRP3 inflammasome induced-pyroptosis, Journal of inflammation (London, England) 17 (2020) 25.

[51] Y. Fang, S. Tian, Y. Pan, W. Li, Q. Wang, Y. Tang, T. Yu, X. Wu, Y. Shi, P. Ma, Y. Shu, Pyroptosis: A new frontier in cancer, Biomed Pharmacother 121 (2020) 109595.

[52] Y. Aachoui, V. Sagulenko, E.A. Miao, K.J. Stacey, Inflammasome-mediated pyroptotic and apoptotic cell death, and defense against infection, Current opinion in microbiology 16(3) (2013) 319-26.

[53] A. de Gassart, F. Martinon, Pyroptosis: Caspase-11 Unlocks the Gates of Death, Immunity 43(5) (2015) 835-7.

[54] X. Chen, J. Cui, X. Zhai, J. Zhang, Z. Gu, X. Zhi, W. Weng, P. Pan, L. Cao, F. Ji, Z. Wang, J. Su, Inhalation of Hydrogen of Different Concentrations Ameliorates Spinal Cord Injury in Mice by Protecting Spinal Cord Neurons from Apoptosis, Oxidative Injury and Mitochondrial Structure Damages, Cellular physiology and biochemistry : international journal of experimental cellular physiology, biochemistry, and pharmacology 47(1) (2018) 176-190.

[55] Z. Qiu, S. Lei, B. Zhao, Y. Wu, W. Su, M. Liu, Q. Meng, B. Zhou, Y. Leng, Z. Xia, NLRP3 Inflammasome Activation-Mediated Pyroptosis Aggravates Myocardial Ischemia/Reperfusion Injury in Diabetic Rats, Oxidative medicine and cellular longevity 2017 (2017) 9743280.

[56] D. Lin, Y. Huang, C. Ho, P. Hung, M. Hsu, T. Wang, T. Wu, T. Lee, Z. Huang, P. Chang, M. Chiang, Oxidative Insults and Mitochondrial DNA Mutation Promote Enhanced Autophagy and Mitophagy Compromising Cell Viability in Pluripotent Cell Model of Mitochondrial Disease, Cells 8(1) (2019).

[57] Q. Li, S. Gao, Z. Kang, M. Zhang, X. Zhao, Y. Zhai, J. Huang, G.Y. Yang, W. Sun, J. Wang, Rapamycin Enhances Mitophagy and Attenuates Apoptosis After Spinal Ischemia-Reperfusion Injury, Front Neurosci $12(2018) 865$. 
[58] Q. Lin, S. Li, N. Jiang, X. Shao, M. Zhang, H. Jin, Z. Zhang, J. Shen, Y. Zhou, W. Zhou, L. Gu, R. Lu, Z. $\mathrm{Ni}$, PINK1-parkin pathway of mitophagy protects against contrast-induced acute kidney injury via decreasing mitochondrial ROS and NLRP3 inflammasome activation, Redox Biol 26 (2019) 101254.

[59] C.T. Chu, Mechanisms of selective autophagy and mitophagy: Implications for neurodegenerative diseases, Neurobiology of disease 122 (2019) 23-34.

[60] D.L. Medina, S. Di Paola, I. Peluso, A. Armani, D. De Stefani, R. Venditti, S. Montefusco, A. ScottoRosato, C. Prezioso, A. Forrester, C. Settembre, W. Wang, Q. Gao, H. Xu, M. Sandri, R. Rizzuto, M.A. De Matteis, A. Ballabio, Lysosomal calcium signalling regulates autophagy through calcineurin and TFEB, Nat Cell Biol 17(3) (2015) 288-99.

[61] C. Settembre, C. Di Malta, V.A. Polito, M. Garcia Arencibia, F. Vetrini, S. Erdin, S.U. Erdin, T. Huynh, D. Medina, P. Colella, M. Sardiello, D.C. Rubinsztein, A. Ballabio, TFEB links autophagy to lysosomal biogenesis, Science (New York, N.Y.) 332(6036) (2011) 1429-33.

[62] N. Raben, R. Puertollano, TFEB and TFE3: Linking Lysosomes to Cellular Adaptation to Stress, Annual review of cell and developmental biology 32 (2016) 255-278.

[63] S. Herzig, R.J. Shaw, AMPK: guardian of metabolism and mitochondrial homeostasis, Nature reviews. Molecular cell biology 19(2) (2018) 121-135.

[64] N.P. Young, A. Kamireddy, J.L. Van Nostrand, L.J. Eichner, M.N. Shokhirev, Y. Dayn, R.J. Shaw, AMPK governs lineage specification through Tfeb-dependent regulation of lysosomes, Genes \& development 30(5) (2016) 535-52.

[65] K. Zhou, H. Chen, J. Lin, H. Xu, H. Wu, G. Bao, J. Li, X. Deng, X. Shui, W. Gao, J. Ding, J. Xiao, H. Xu, FGF21 augments autophagy in random-pattern skin flaps via AMPK signaling pathways and improves tissue survival, Cell death \& disease 10(12) (2019) 872.

[66] H.J. Shin, H. Kim, S. Oh, J.G. Lee, M. Kee, H.J. Ko, M.N. Kweon, K.J. Won, S.H. Baek, AMPK-SKP2CARM1 signalling cascade in transcriptional regulation of autophagy, Nature 534(7608) (2016) 553-7.

[67] T. Gong, Y. Yang, T. Jin, W. Jiang, R. Zhou, Orchestration of NLRP3 Inflammasome Activation by lon Fluxes, Trends in immunology 39(5) (2018) 393-406.

[68] Y. Wang, L. Jia, Cathepsin B aggravates coxsackievirus B3-induced myocarditis through activating the inflammasome and promoting pyroptosis, 14(1) (2018) e1006872.

\section{Figures}




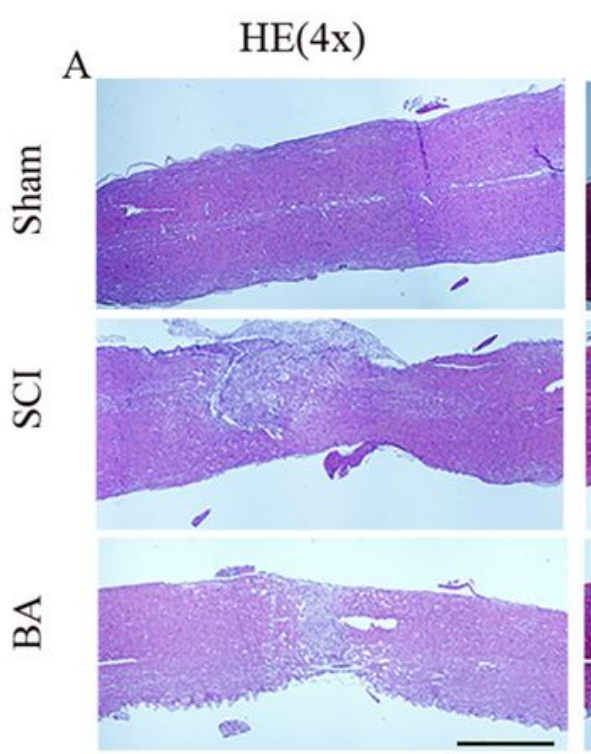

C

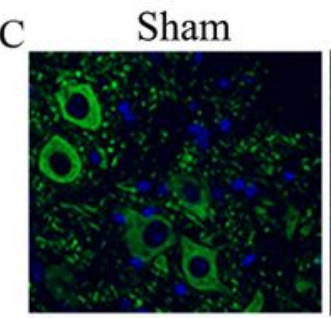

E

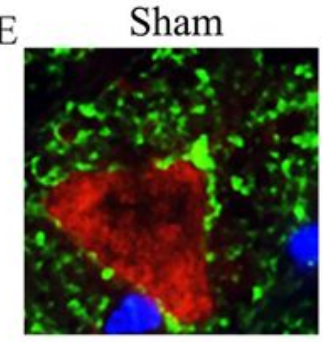

G

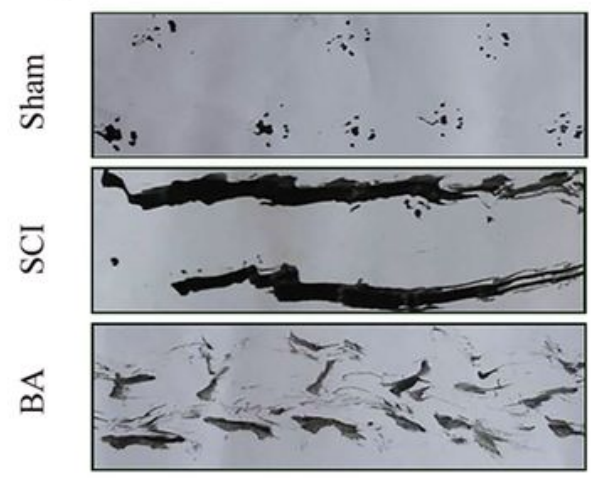

SCI
$\operatorname{Masson}(4 \mathrm{x})$
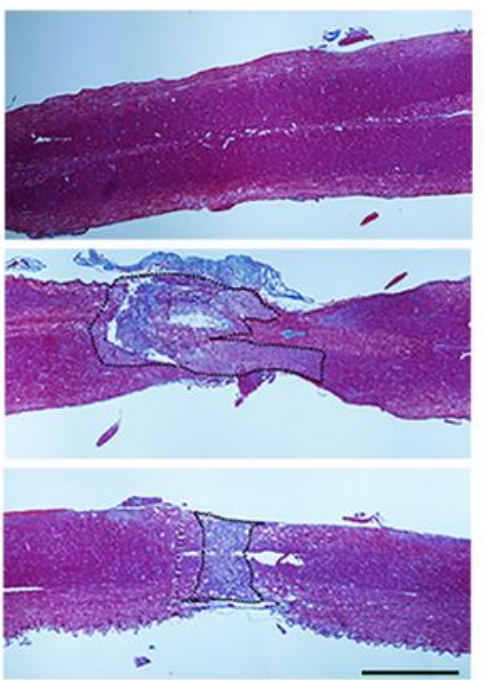

BA
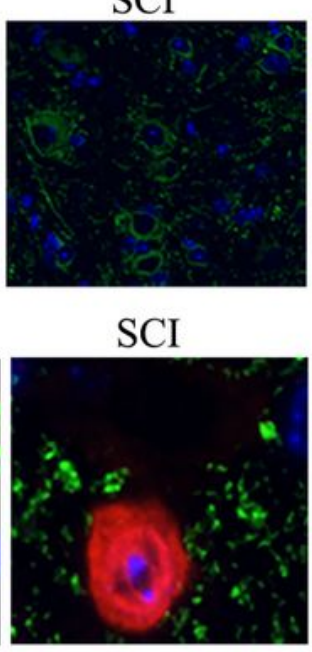

$\mathrm{H}$

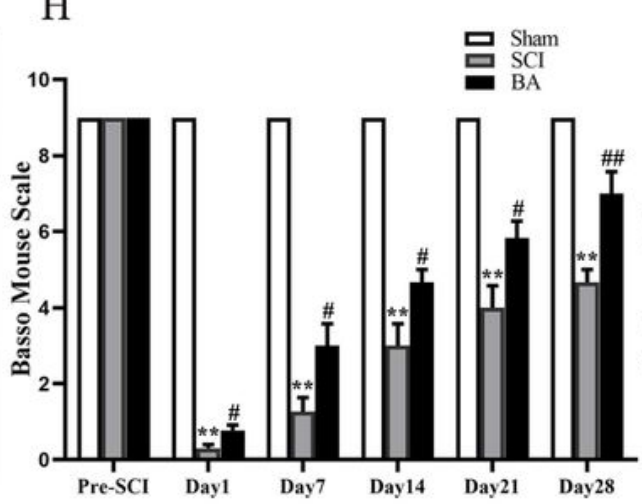

B
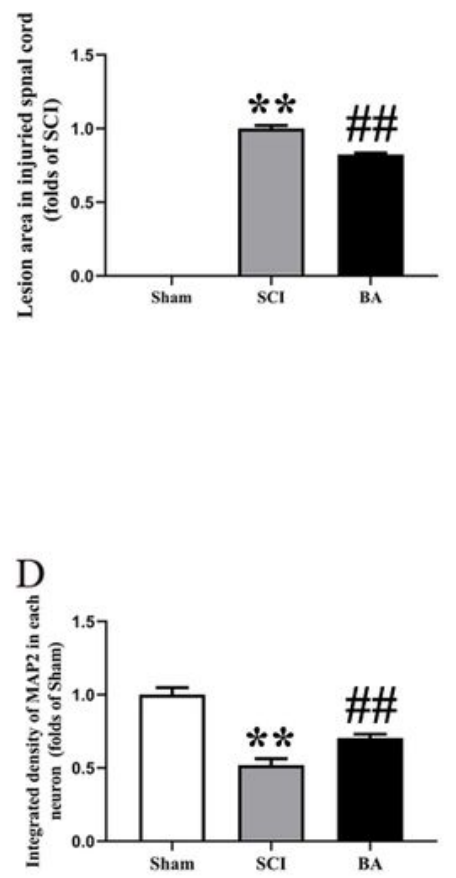

$\mathrm{F}$

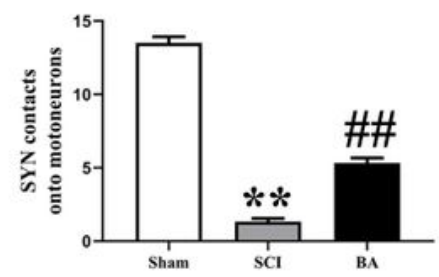

L

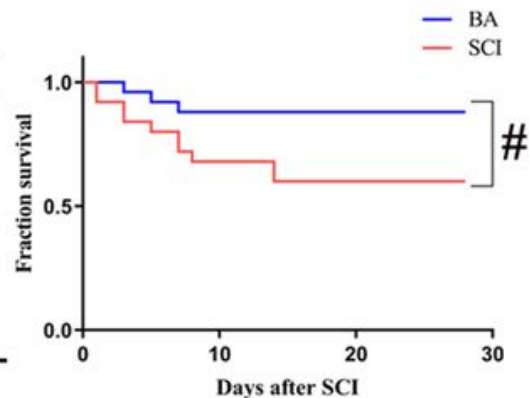

\section{Figure 1}

BA promotes functional recovery after SCl. (A) Longitudinal spinal cord sections from the indicated groups at day 14 were analyzed by HE staining and Masson staining (scale bar=1000 $\mu \mathrm{m})$. (B) Quantitative analysis of Masson positive lesions in the spinal cords of each group. (C) Images (30x) of the spinal cord sections in each group stained with antibodies against MAP2 (scale bar $=25 \mu \mathrm{m})$. (D) Quantification data from (C) showing the optical density of MAP2 in the injured spinal cord at day 28. (E) 
Images (150x) of spinal cord sections below the injury (T11-T12) stained at day 28 with antibodies

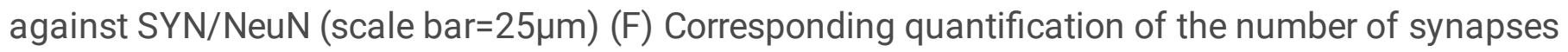
contacting motor neurons. (G) Photos of mice footprints at day 28 after SCl. (H) Basso mouse scale (BMS) for the indicated groups and time points, $n=(6-12)$. (I) The analysis of survival rate of $\mathrm{SCl}$ group and $\mathrm{BA}$ group subjected to $\mathrm{SCl}$ at days $1,7,14,21$ and 28 . The values are expressed as the means $\pm \mathrm{SEM}$, $\mathrm{n}=6$ per group (except for BMS). ${ }^{*} \mathrm{p}<<0.01$, vs. Sham group. $\# p<0.05$ and $\# \# p<0.01$, vs. SCl group.

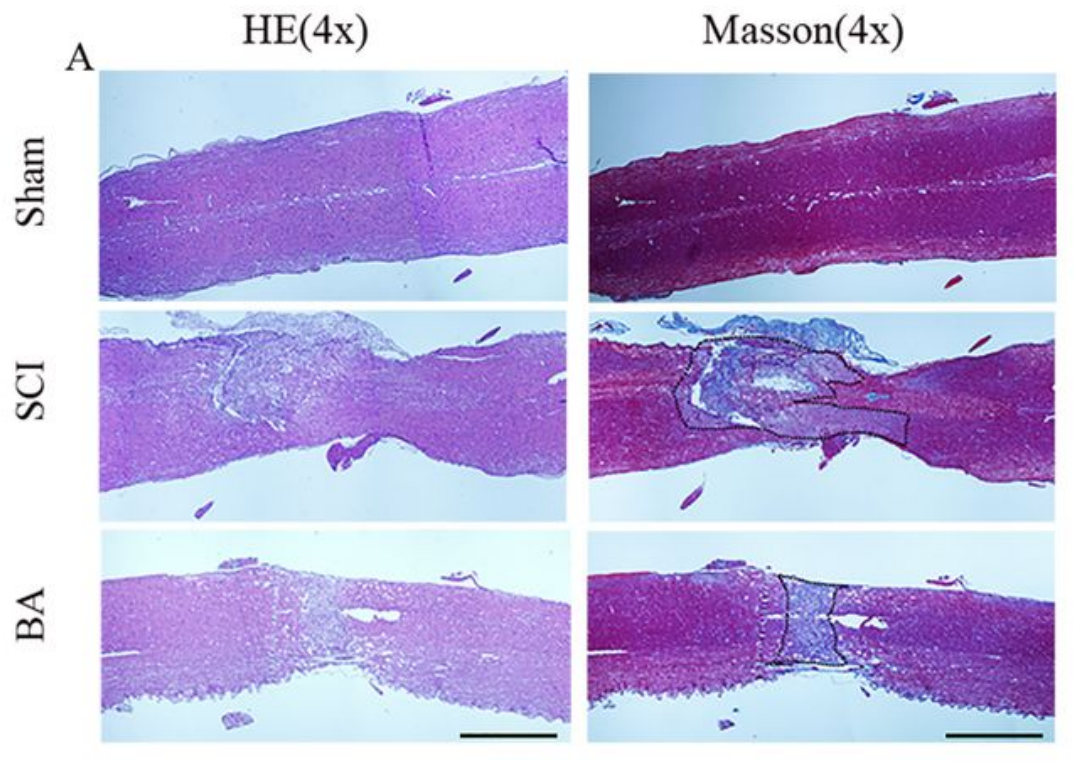

B

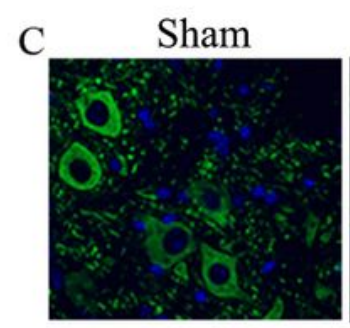

$\mathrm{E}$

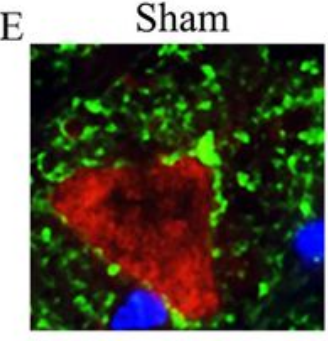

G

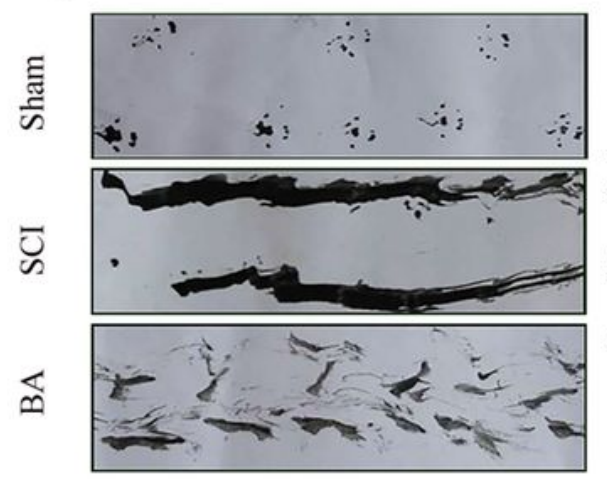

$\mathrm{SCI}$

SCI
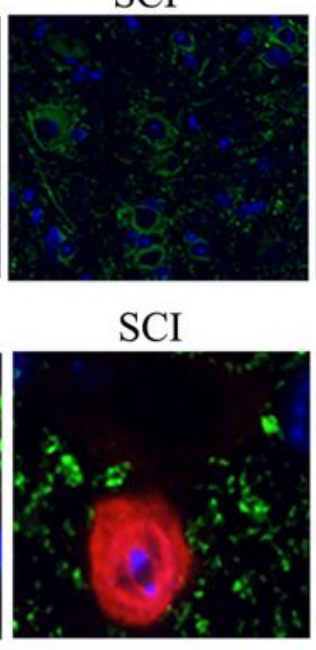

$\mathrm{H}$

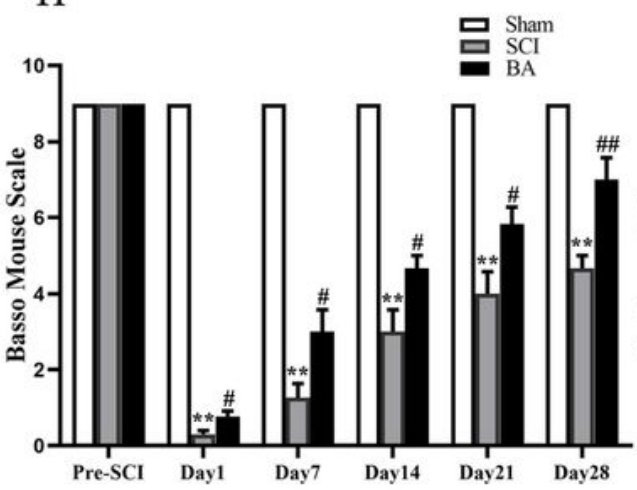

BA

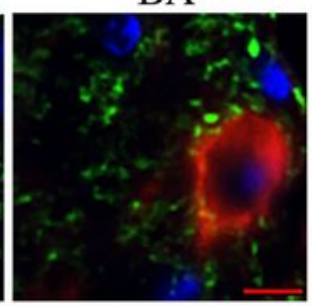

F
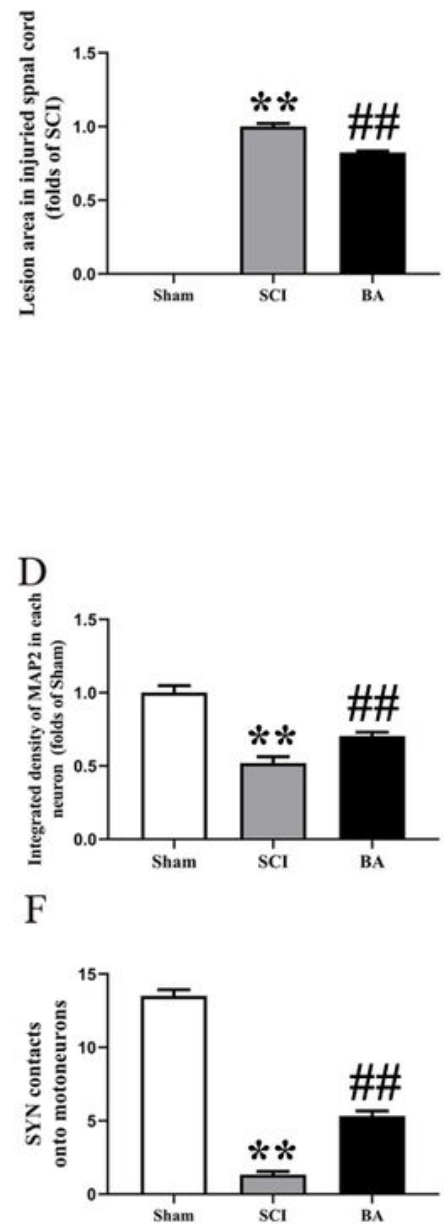

L

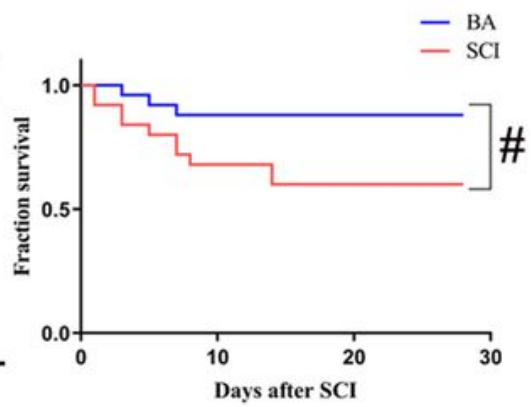

Figure 1 
BA promotes functional recovery after $\mathrm{SCl}$. (A) Longitudinal spinal cord sections from the indicated groups at day 14 were analyzed by HE staining and Masson staining (scale bar=1000 $\mu \mathrm{m}$ ). (B) Quantitative analysis of Masson positive lesions in the spinal cords of each group. (C) Images (30x) of the spinal cord sections in each group stained with antibodies against MAP2 (scale bar= $25 \mu \mathrm{m})$. (D) Quantification data from (C) showing the optical density of MAP2 in the injured spinal cord at day 28. (E) Images (150x) of spinal cord sections below the injury (T11-T12) stained at day 28 with antibodies

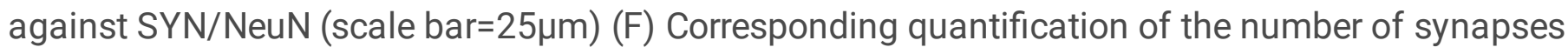
contacting motor neurons. (G) Photos of mice footprints at day 28 after SCl. (H) Basso mouse scale (BMS) for the indicated groups and time points, $n=(6-12)$. (I) The analysis of survival rate of $\mathrm{SCl}$ group and $\mathrm{BA}$ group subjected to $\mathrm{SCl}$ at days $1,7,14,21$ and 28 . The values are expressed as the means $\pm \mathrm{SEM}$, $n=6$ per group (except for BMS). ${ }^{\star \star} p<0.01$, vs. Sham group. $\# p<0.05$ and $\# \# p<0.01$, vs. $S C l$ group. 

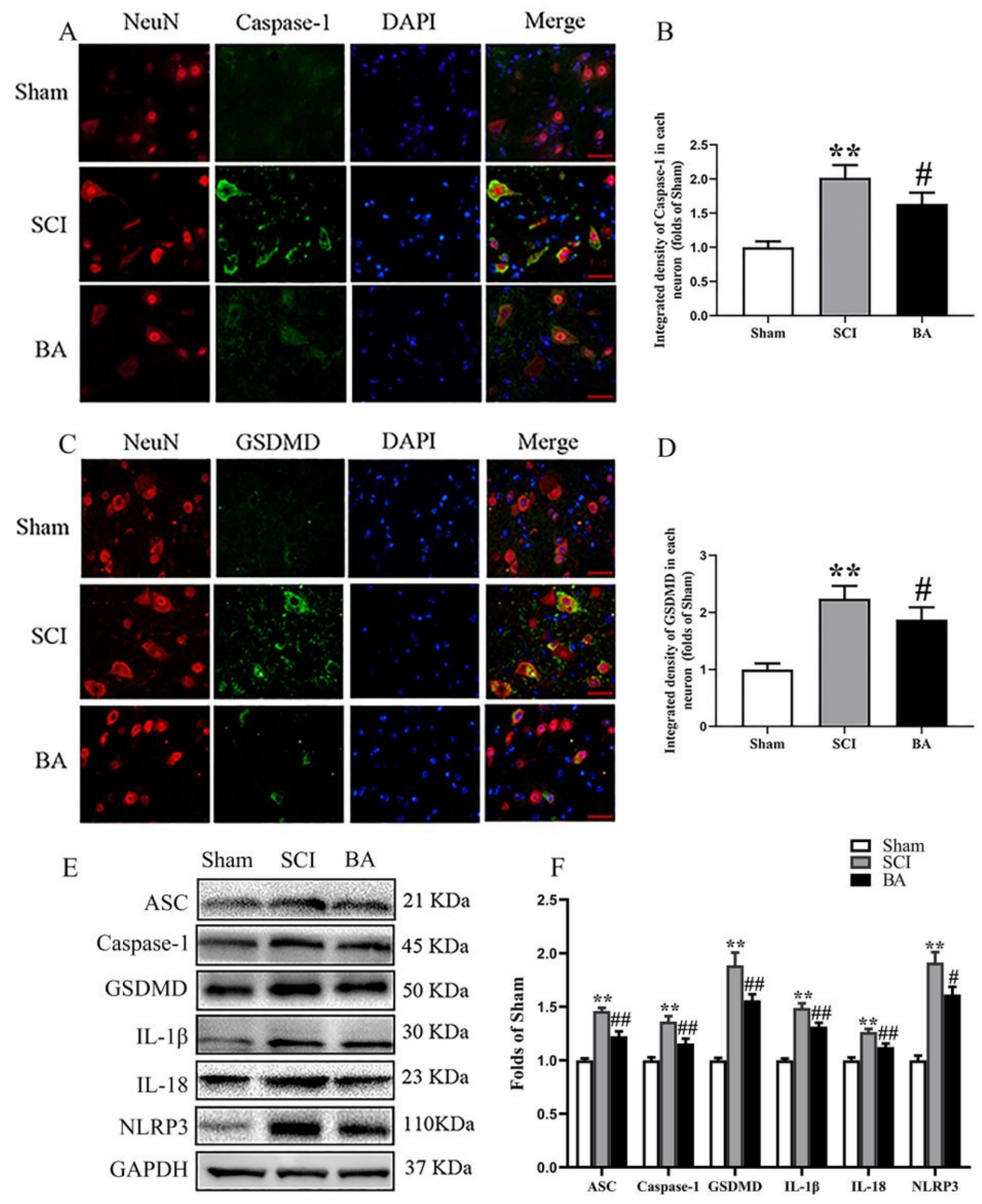

\section{Figure 2}

BA attenuates pyroptosis after SCl. (A) Immunofluorescence staining for Caspase-1 and NeuN colocalization in the spinal cords of the Sham, SCl, and BA groups (Scan bar= $25 \mu \mathrm{m}$ ) (B) The quantitative mean optical density of the Caspase-1 in motor neurons of spinal cord lesion. (C) Immunofluorescence staining for GSDMD and NeuN co-localization in the spinal cords of the Sham, SCl, and BA groups (Scan bar $=25 \mu \mathrm{m})(\mathrm{D})$ The quantitative mean optical density of the GSDMD in motor neurons of spinal cord 
lesion. (E)Western blotting for ASC, Caspase-1, GSDMD, IL-1 $\beta$, IL-18 and NLRP3 expression levels in the Sham, $\mathrm{SCl}$, and BA groups. The gels were run under the same experimental conditions, and the cropped blots are shown here. (F) The optical density values of the ASC, Caspase-1, GSDMD, IL-1 1 , IL-18 and NLRP3 expression levels were quantified and analyzed in each group. The values are expressed as the means $\pm S E M, n=6$ per group. ${ }^{*} p<0.01$, vs. Sham group. $\# p<0.05$ and $\# \# p<0.01$, vs. $S C l$ group.
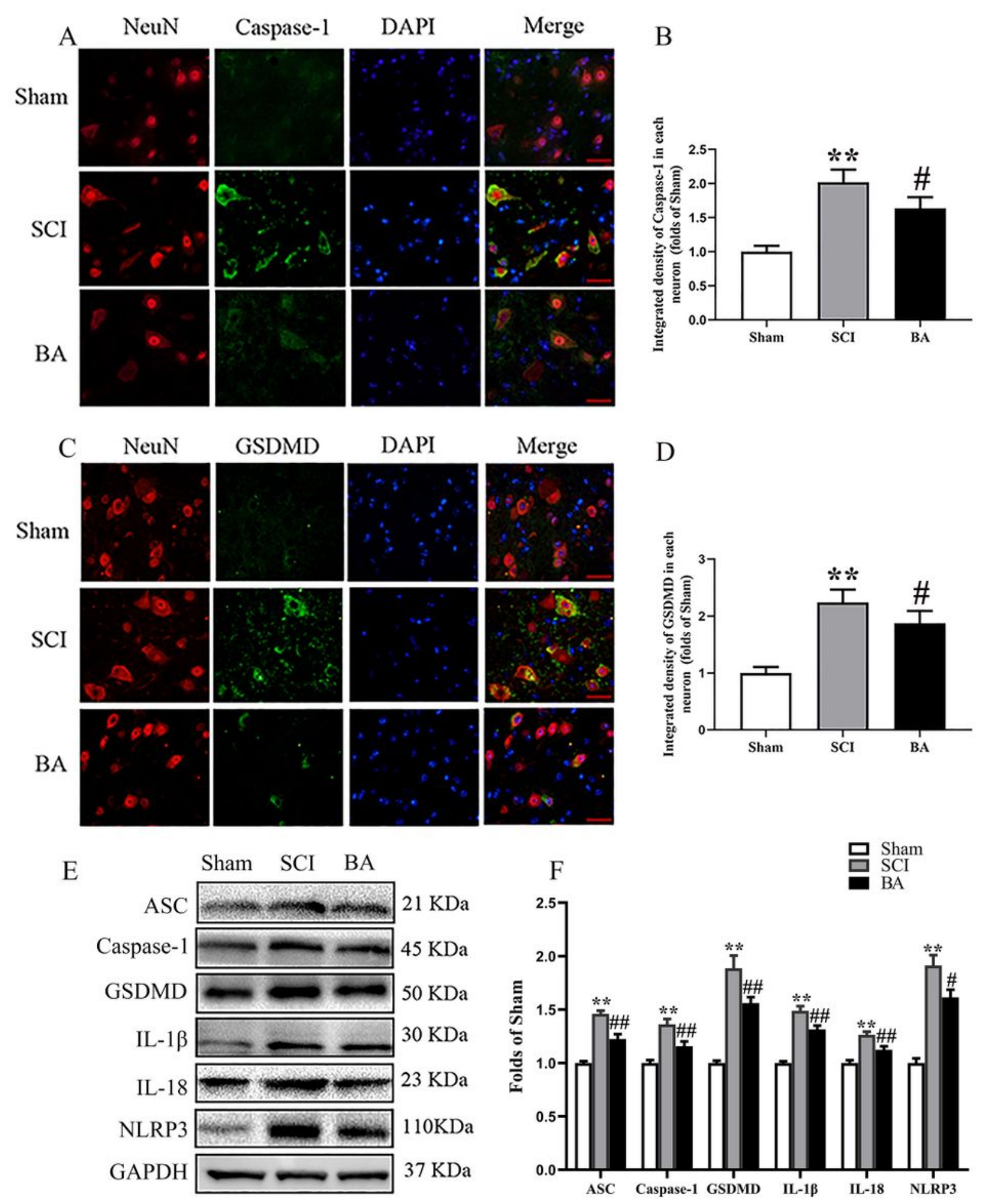

Figure 2 
BA attenuates pyroptosis after SCl. (A) Immunofluorescence staining for Caspase-1 and NeuN colocalization in the spinal cords of the Sham, $\mathrm{SCl}$, and BA groups (Scan bar= $25 \mu \mathrm{m})(\mathrm{B})$ The quantitative mean optical density of the Caspase-1 in motor neurons of spinal cord lesion. (C) Immunofluorescence staining for GSDMD and NeuN co-localization in the spinal cords of the Sham, SCl, and BA groups (Scan bar $=25 \mu \mathrm{m})(\mathrm{D})$ The quantitative mean optical density of the GSDMD in motor neurons of spinal cord lesion. (E)Western blotting for ASC, Caspase-1, GSDMD, IL-1 $\beta$, IL-18 and NLRP3 expression levels in the Sham, $\mathrm{SCl}$, and $\mathrm{BA}$ groups. The gels were run under the same experimental conditions, and the cropped blots are shown here. (F) The optical density values of the ASC, Caspase-1, GSDMD, IL-1 $1 \beta$, IL-18 and NLRP3 expression levels were quantified and analyzed in each group. The values are expressed as the means $\pm S E M, n=6$ per group. ${ }^{*} p<0.01$, vs. Sham group. $\# p<0.05$ and $\# \# p<0.01$, vs. $S C l$ group. 


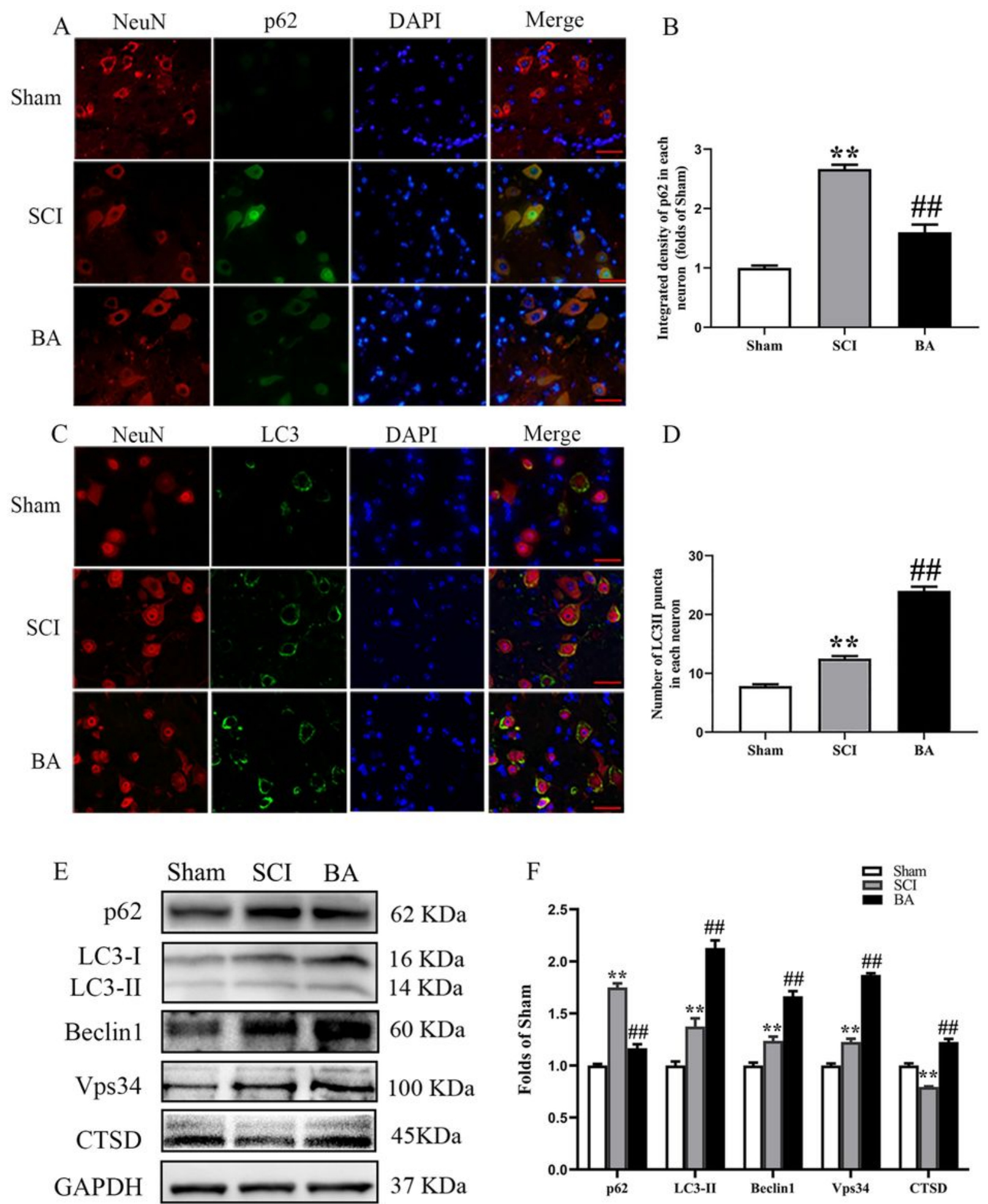

\section{Figure 3}

BA enhances autophagy after SCl. (A) Immunofluorescence staining for p62 and NeuN co-localization at the spinal cord lesion after SCl (Scan bar=25 $\mathrm{mm}$ ). (B) The quantitative mean optical density of the p62 in motor neurons of spinal cord lesion in each group. (C) Immunofluorescence staining for LC3II and NeuN co-localization at the spinal cord lesion after SCl (Scan bar= $25 \mu \mathrm{m})$. (D)The quantitative mean number of the LC3II positive neurons in motor neurons of spinal cord lesion in each group. (E) Western blotting for 
p62, LC3II, Beclin1, Vps34, and CTSD expression levels in the Sham, SCI and BA groups. The gels were run under the same experimental conditions, and the cropped blots are shown here. (F) The optical density values of the p62, LC3II, Beclin1, Vps34, and CTSD expression levels were quantified and analyzed in each group. The values are expressed as the means $\pm S E M, n=6$ per group. ${ }^{*} p<0.01$, vs. Sham group. \#\#p<0.01, vs. SCl group.
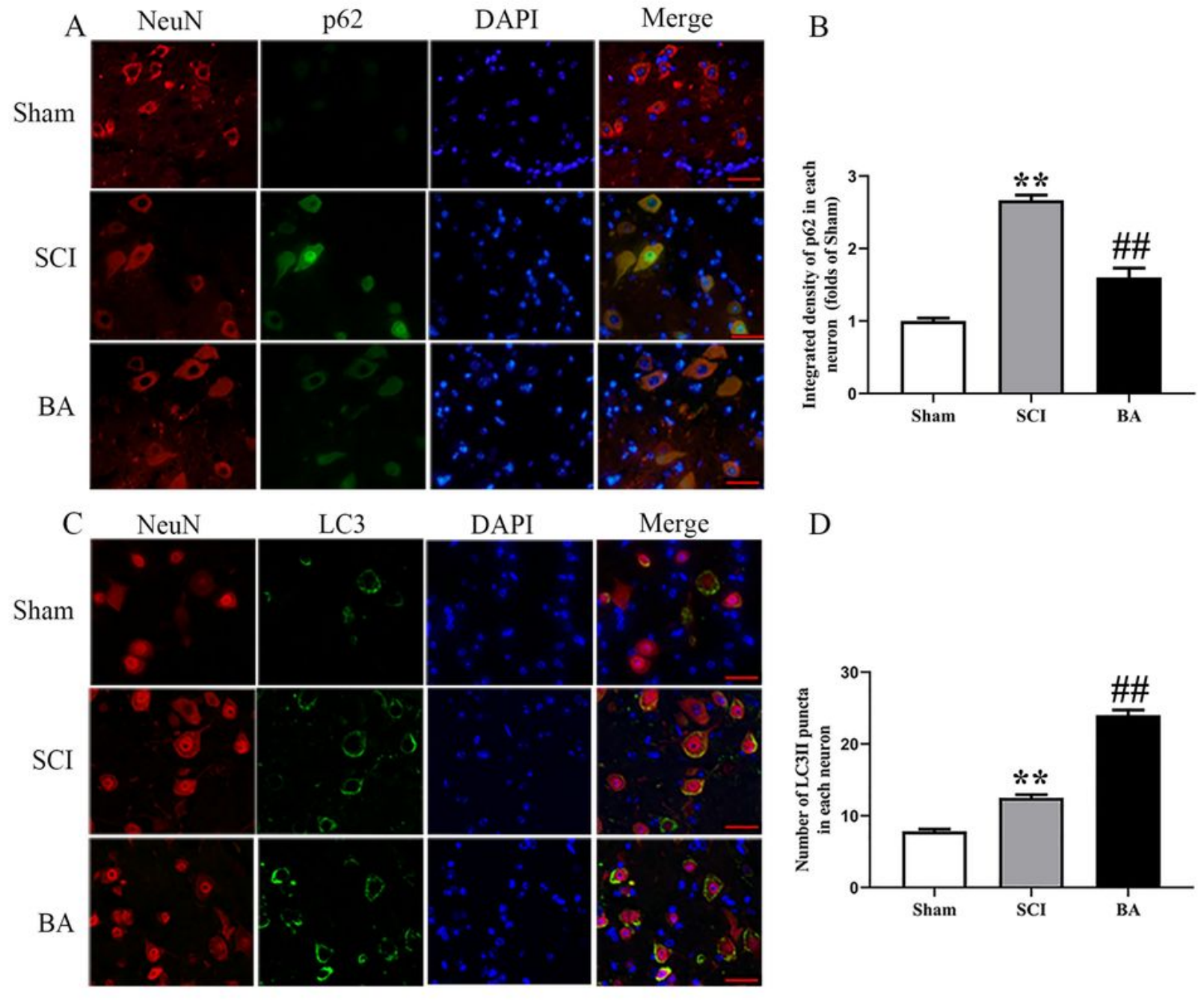

$\mathrm{D}$
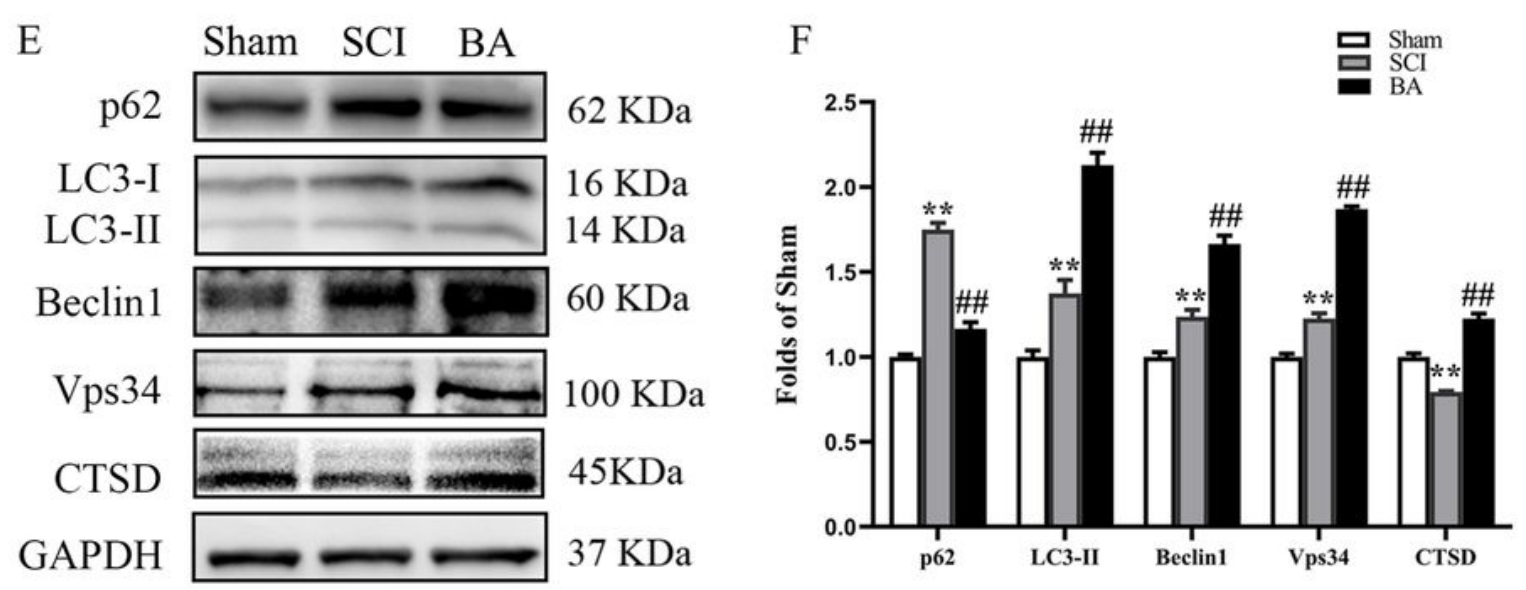

Figure 3 
BA enhances autophagy after SCl. (A) Immunofluorescence staining for p62 and NeuN co-localization at

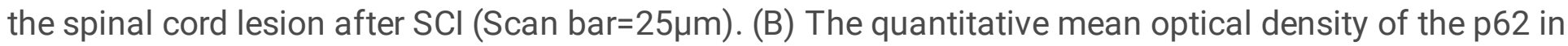
motor neurons of spinal cord lesion in each group. (C) Immunofluorescence staining for LC3II and NeuN co-localization at the spinal cord lesion after SCI (Scan bar= 25 $\mu \mathrm{m}$ ). (D)The quantitative mean number of the LC3II positive neurons in motor neurons of spinal cord lesion in each group. (E) Western blotting for p62, LC3II, Beclin1, Vps34, and CTSD expression levels in the Sham, SCI and BA groups. The gels were run under the same experimental conditions, and the cropped blots are shown here. $(F)$ The optical density values of the p62, LC3II, Beclin1, Vps34, and CTSD expression levels were quantified and analyzed in each group. The values are expressed as the means $\pm S E M, n=6$ per group. ${ }^{\star \star} p<0.01$, vs. Sham group. \#\#p<0.01, vs. SCl group.
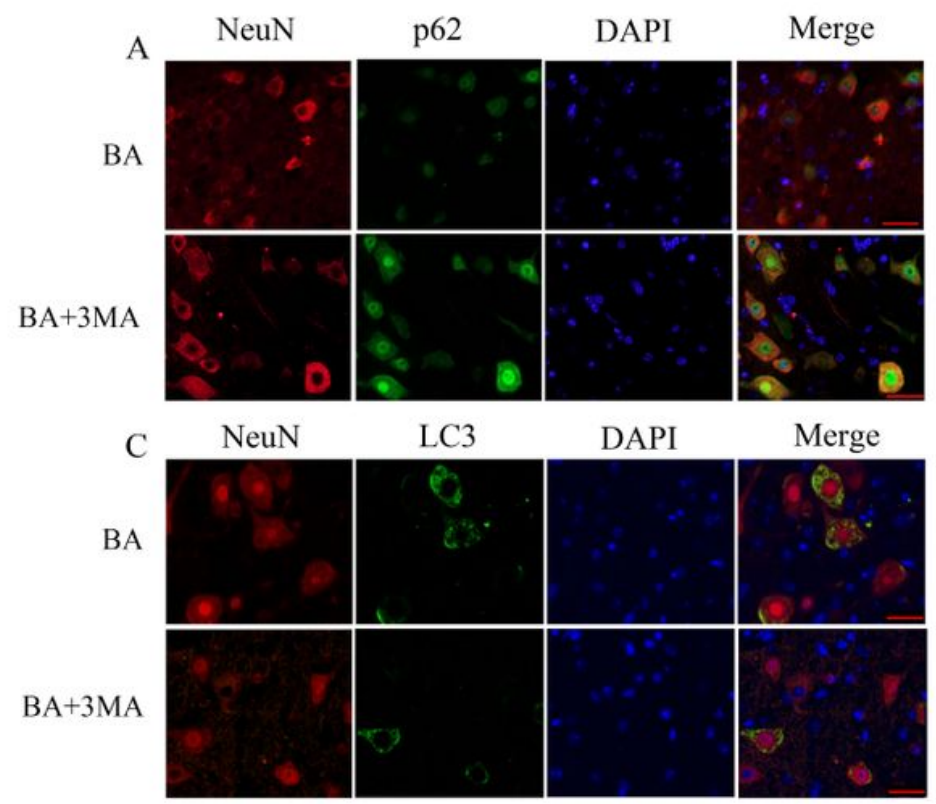

DAPI
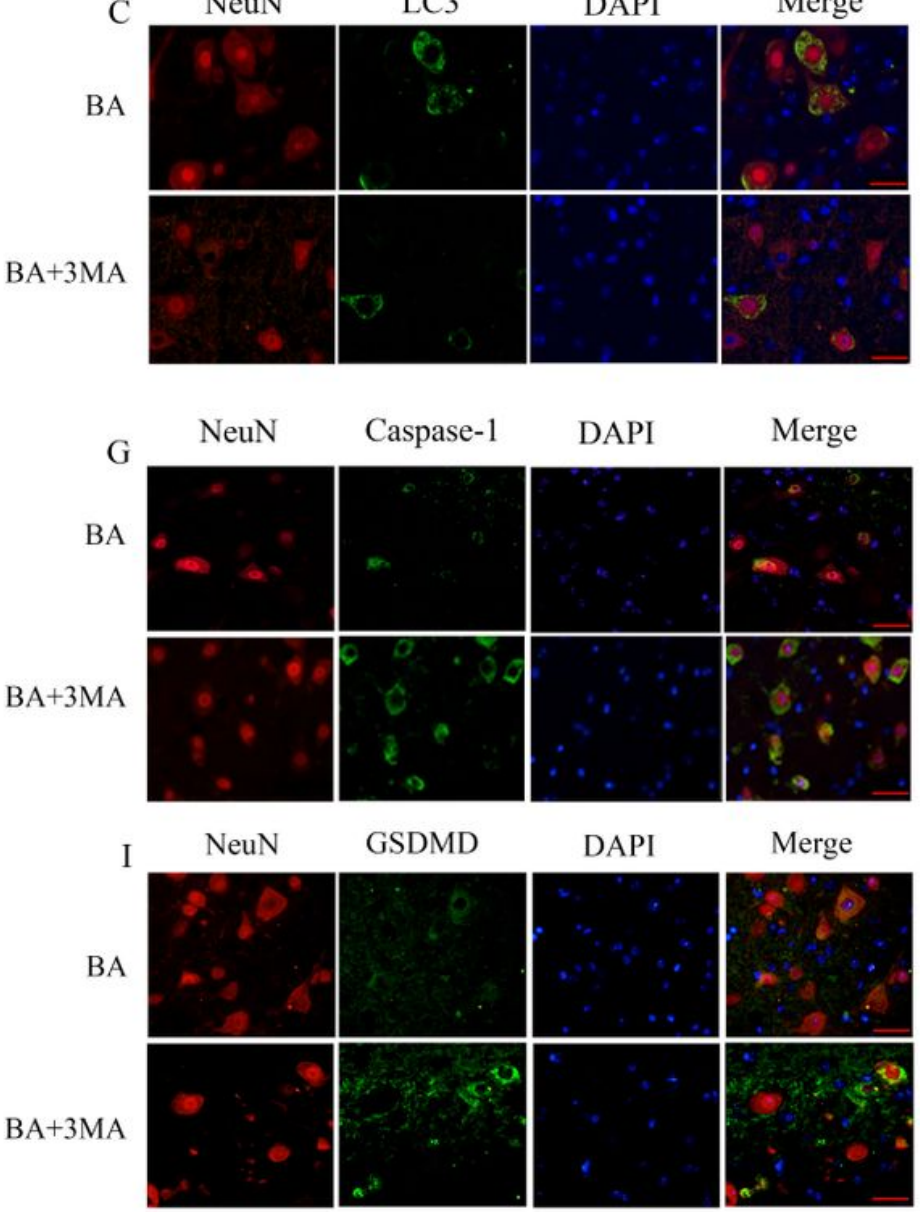

B

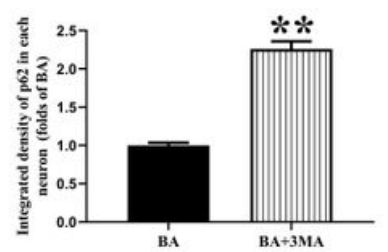

D
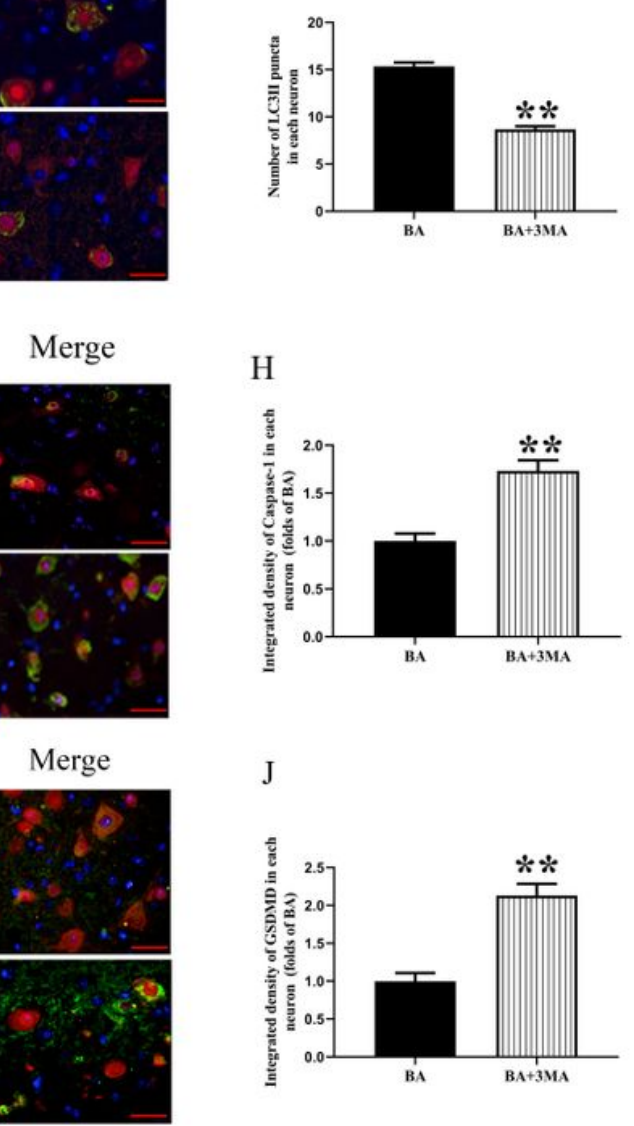
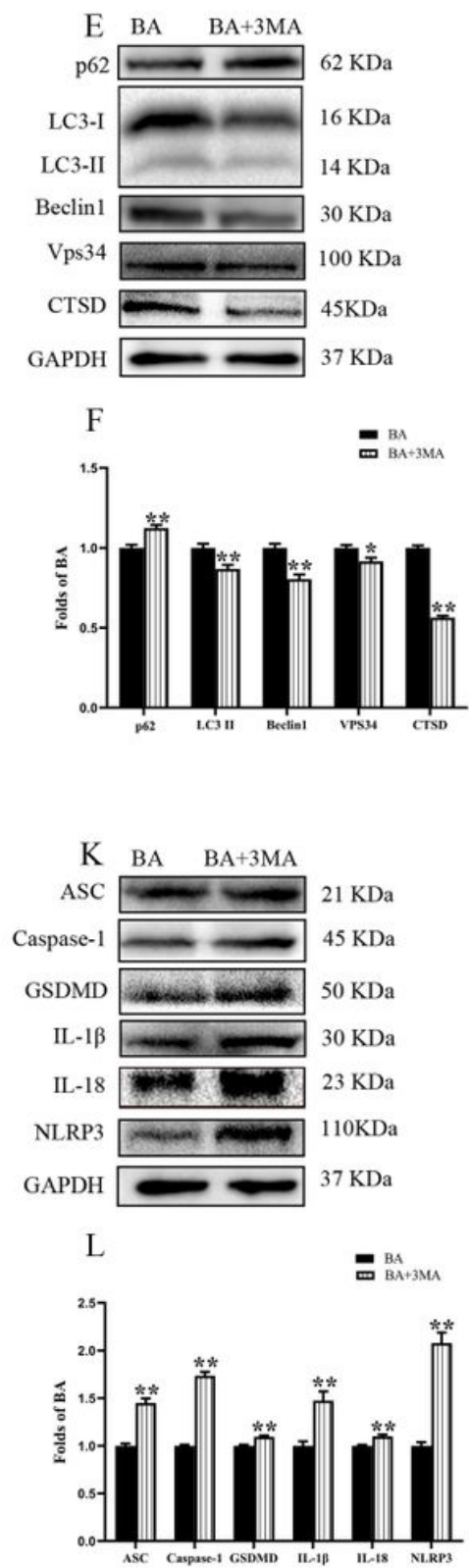

Figure 4 
Inhibition of autophagy reverses the effects of BA on pyroptosis after SCl. (A) Immunofluorescence

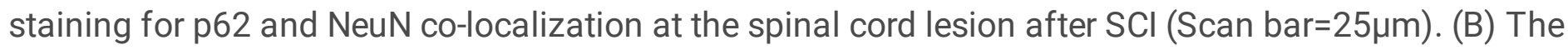
quantitative mean optical density of the p62 in motor neurons of spinal cord lesion in each group. (C) Immunofluorescence staining for LC3II and NeuN co-localization at the spinal cord lesion after SCI (Scan bar= $25 \mu \mathrm{m}$ ). (D) The quantitative mean number of the LC3II positive neurons in motor neurons of spinal cord lesion in each group. (E) Western blotting for the p62, LC3II, Beclin1, Vps34, and CTSD expression levels in the BA and BA+3MA groups. The gels were run under the same experimental conditions, and the cropped blots are shown here. (F) The optical density values of the p62, LC3II, Beclin1, Vps34, and CTSD expression levels were quantified and analyzed in each group. (G) Immunofluorescence staining for Caspase-1 and NeuN co-localization in the spinal cords of the BA and BA+3MA groups (Scan bar= $25 \mu \mathrm{m}$ ) $(H)$ The quantitative mean optical density of the Caspase-1 in motor neurons of spinal cord lesion. (I) Immunofluorescence staining for GSDMD and NeuN co-localization in the spinal cords of the BA and BA+3MA groups (Scan bar $=25 \mu \mathrm{m})(\mathrm{J})$ The quantitative mean optical density of the GSDMD in motor neurons of spinal cord lesion. (K) Western blotting for the ASC, Caspase-1, GSDMD, IL-1 $\beta$, IL-18 and NLRP3 expression levels in the BA and BA+3MA groups. The gels were run under the same experimental conditions, and the cropped blots are shown here. (L) The optical density values of the ASC, Caspase-1, GSDMD, IL-1 1 , IL-18 and NLRP3 expression levels were quantified and analyzed in each group. The values are expressed as the means \pm SEM, $n=6$ per group. ${ }^{*} p<0.05$ and ${ }^{* \star} p<0.01$, vs. BA group. 

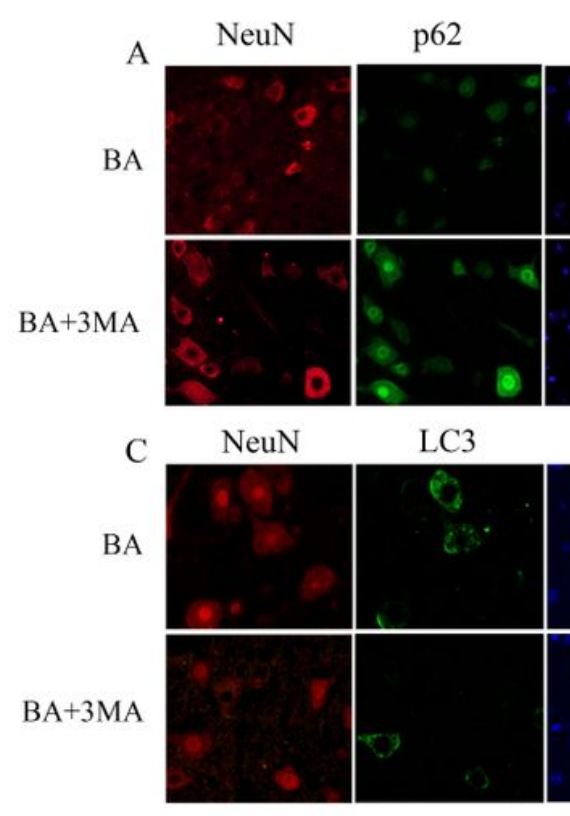

LC3
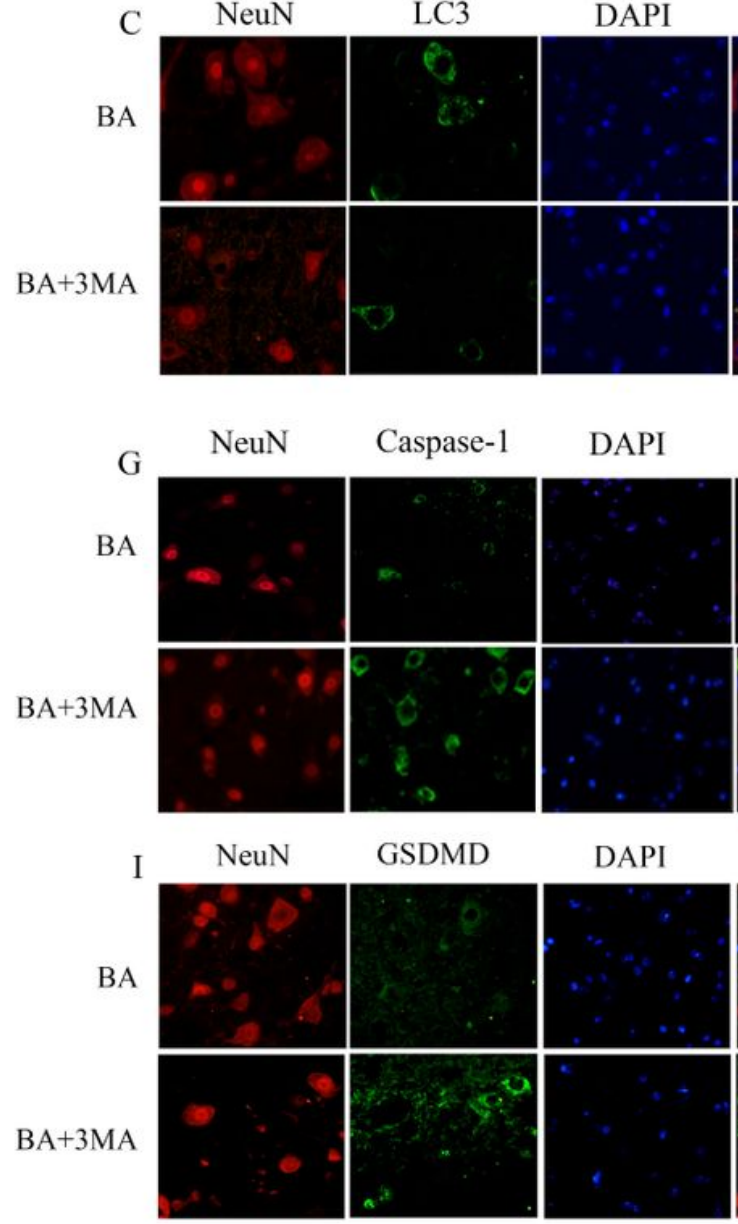

Merge
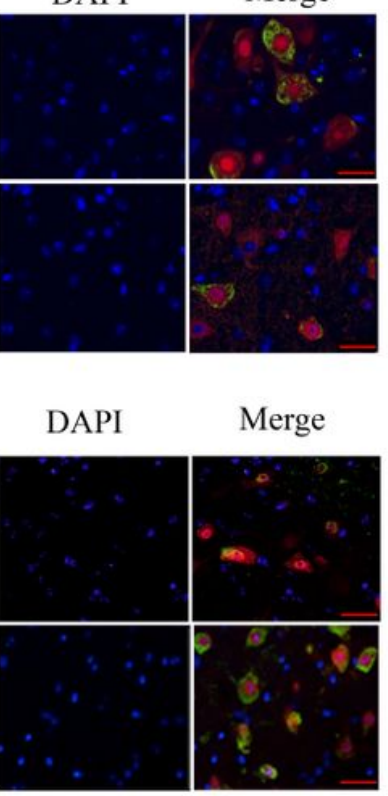

DAPI
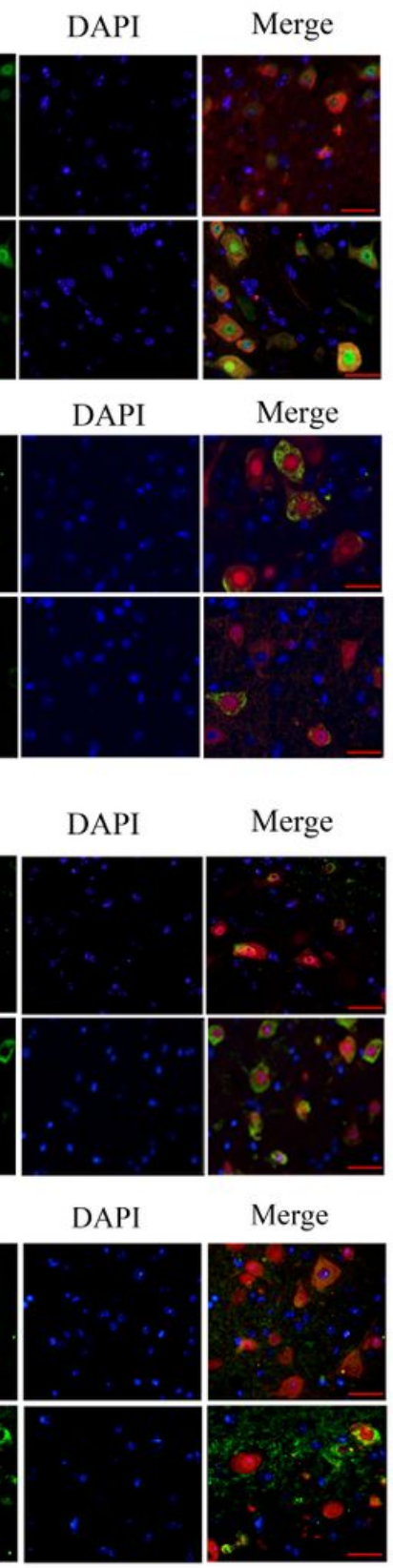

B

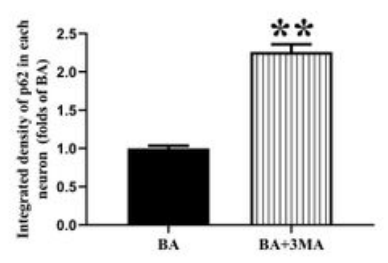

D
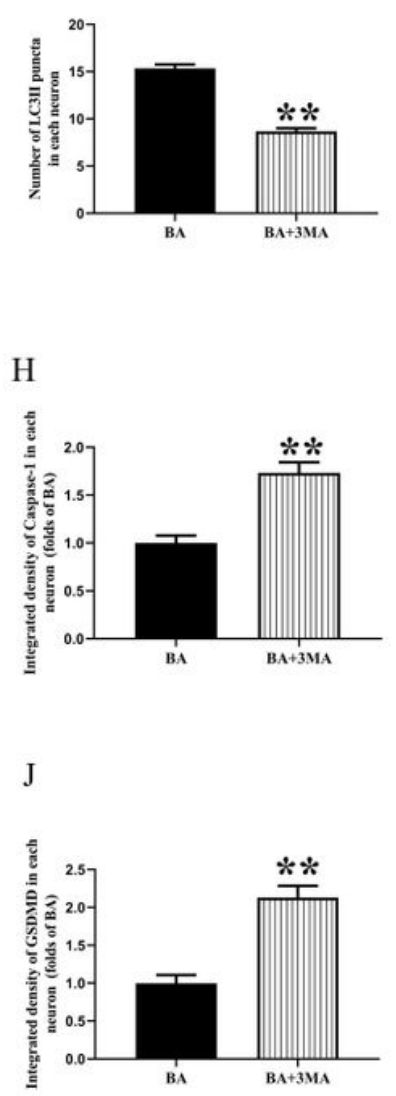
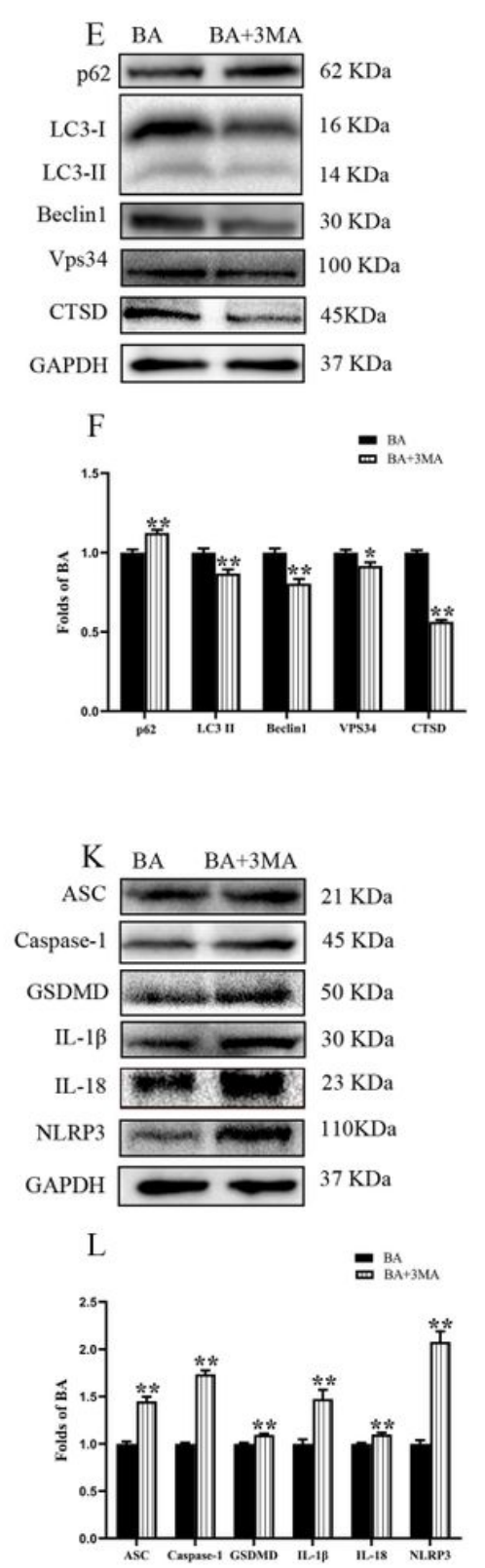

\section{Figure 4}

Inhibition of autophagy reverses the effects of BA on pyroptosis after SCl. (A) Immunofluorescence staining for p62 and NeuN co-localization at the spinal cord lesion after SCl (Scan bar=25 $\mu \mathrm{m}$ ). (B) The quantitative mean optical density of the p62 in motor neurons of spinal cord lesion in each group. (C) Immunofluorescence staining for LC3II and NeuN co-localization the spinal cord lesion after SCI (Scan bar $=25 \mu \mathrm{m})$. (D) The quantitative mean number of the LC3II positive neurons in motor neurons of spinal cord lesion in each group. (E) Western blotting for the p62, LC3II, Beclin1, Vps34, and CTSD expression levels in the BA and BA+3MA groups. The gels were run under the same experimental conditions, and the cropped blots are shown here. (F) The optical density values of the p62, LC3II, Beclin1, Vps34, and CTSD expression levels were quantified and analyzed in each group. (G) Immunofluorescence staining for Caspase- 1 and NeuN co-localization in the spinal cords of the BA and BA+3MA groups (Scan bar $=25 \mu \mathrm{m}$ ) 
$(\mathrm{H})$ The quantitative mean optical density of the Caspase-1 in motor neurons of spinal cord lesion. (I) Immunofluorescence staining for GSDMD and NeuN co-localization in the spinal cords of the BA and BA+3MA groups (Scan bar $=25 \mu \mathrm{m})(\mathrm{J})$ The quantitative mean optical density of the GSDMD in motor neurons of spinal cord lesion. (K) Western blotting for the ASC, Caspase-1, GSDMD, IL-1 $\beta, I L-18$ and NLRP3 expression levels in the BA and BA+3MA groups. The gels were run under the same experimental conditions, and the cropped blots are shown here. (L) The optical density values of the ASC, Caspase-1, GSDMD, IL-1 $\beta$, IL-18 and NLRP3 expression levels were quantified and analyzed in each group. The values are expressed as the means $\pm S E M, n=6$ per group. ${ }^{*} p<0.05$ and ${ }^{*} p<0.01$, vs. BA group.
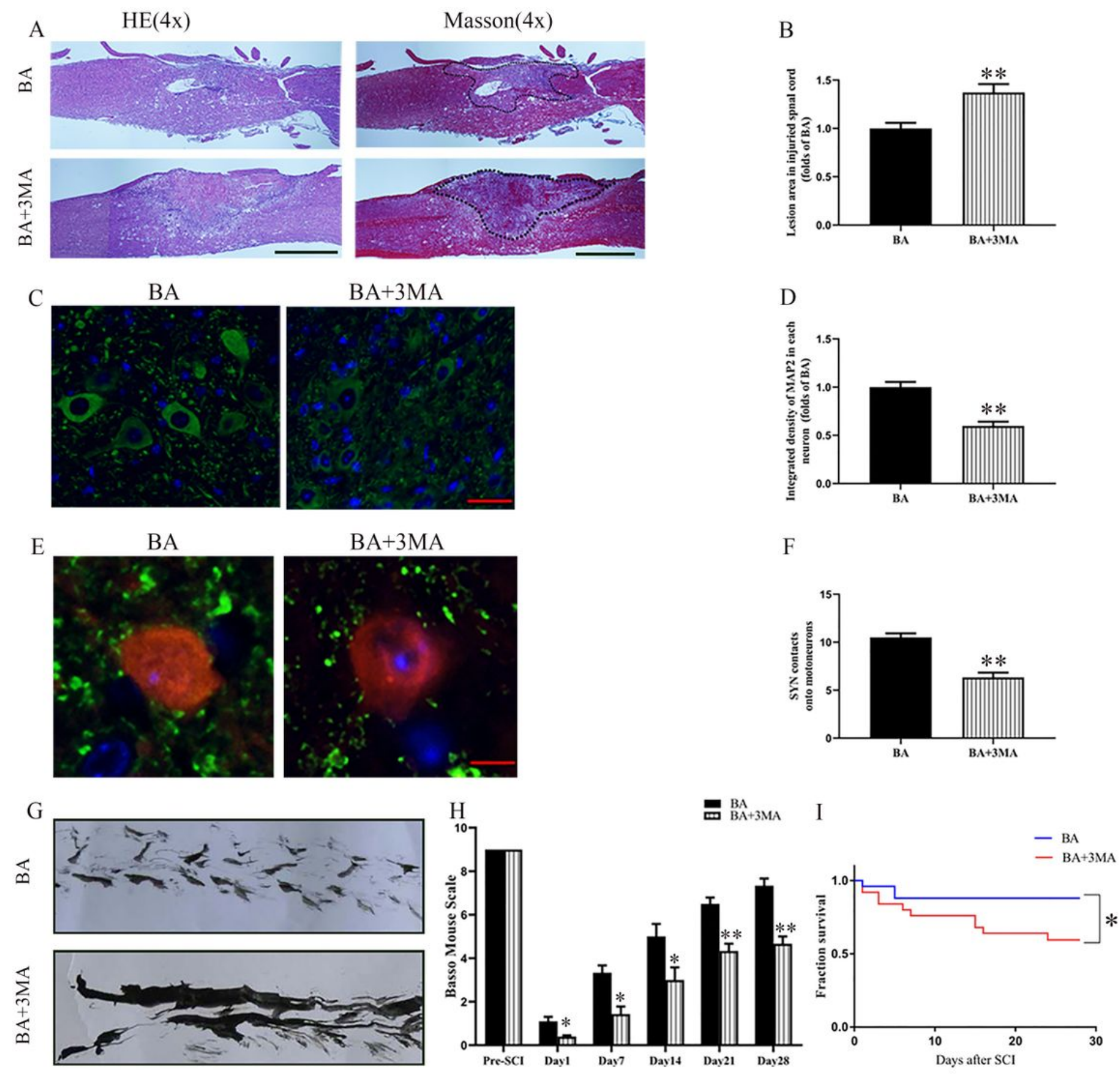

Figure 5 
Inhibition of autophagy reverses the effects of BA on functional recovery after SCl. (A) Longitudinal spinal cord sections from the indicated groups at day 14 were analyzed by HE staining and Masson staining (scale bar=1000 $\mu \mathrm{m}$ ). (B) Quantitative analysis of Masson positive lesions in the spinal cords of each group. (C) Images (30x) of the spinal cord sections in each group stained with antibodies against MAP2 (scale bar=25 $\mu \mathrm{m}$ ). (D) Quantification data from (C) showing the optical density of MAP2 in the injured spinal cord at day 28. (E) Images (150x) of spinal cord sections below the injury (T11-T12) stained at day 28 with antibodies against SYN/NeuN (scale bar $=5 \mu \mathrm{m}$ ). (F) Corresponding quantification of the number of synapses contacting motor neurons. (G) Photos of mice footprints at day 28 after SCl. (H) Basso mouse scale (BMS) for the indicated groups and time points, $n=(6-12)$. (I) The analysis of survival rate of $B A$ mice and $B A+3 M A$ mice subjected to $S C l$ at days $1,7,14,21$, and 28 . The values are expressed as the means \pm SEM, $n=6$ per group (except for BMS). ${ }^{*} p<0.05$ and ${ }^{* *} p<0.01$, vs. BA group.
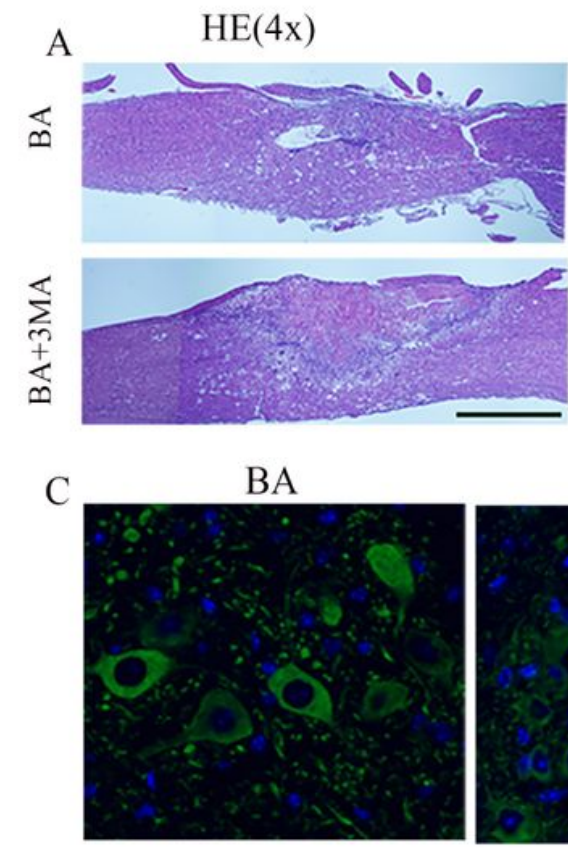

E

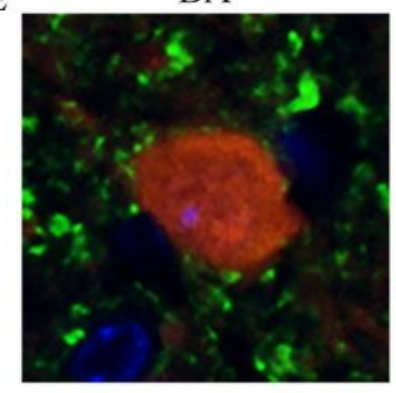

G
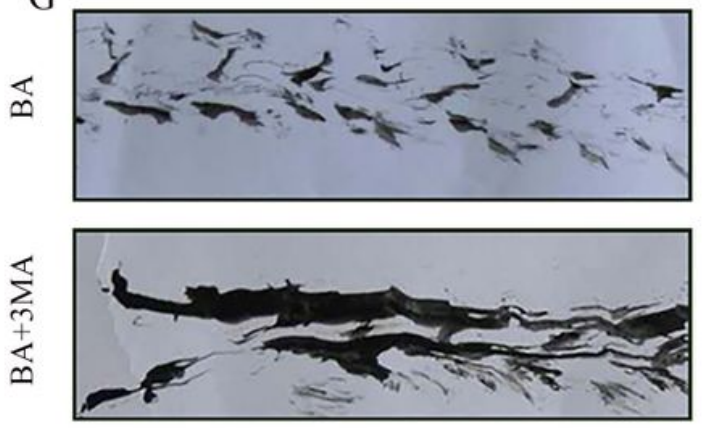

$\operatorname{Masson}(4 \mathrm{x})$

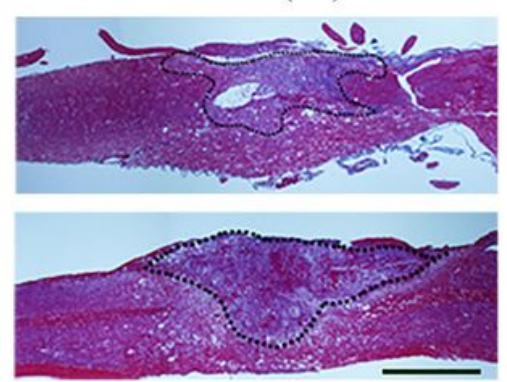

$\mathrm{BA}+3 \mathrm{MA}$

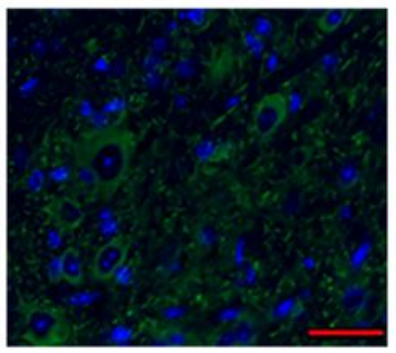

$\mathrm{BA}+3 \mathrm{MA}$
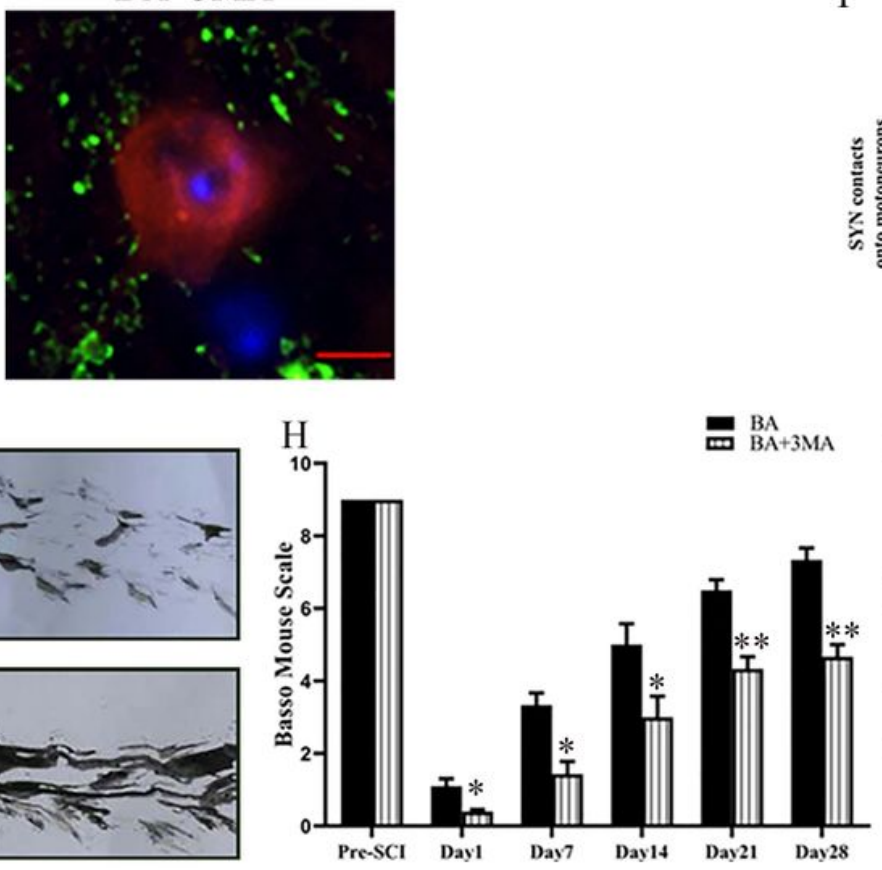

Page $32 / 44$
B

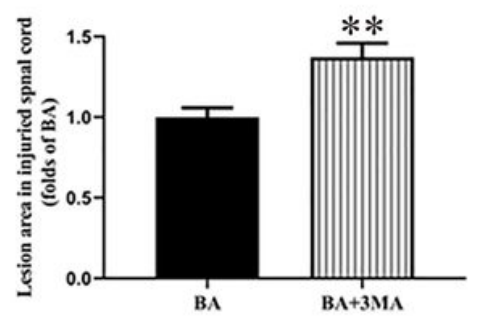

$\mathrm{D}$

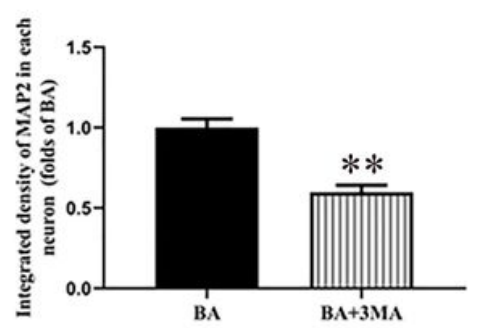

F
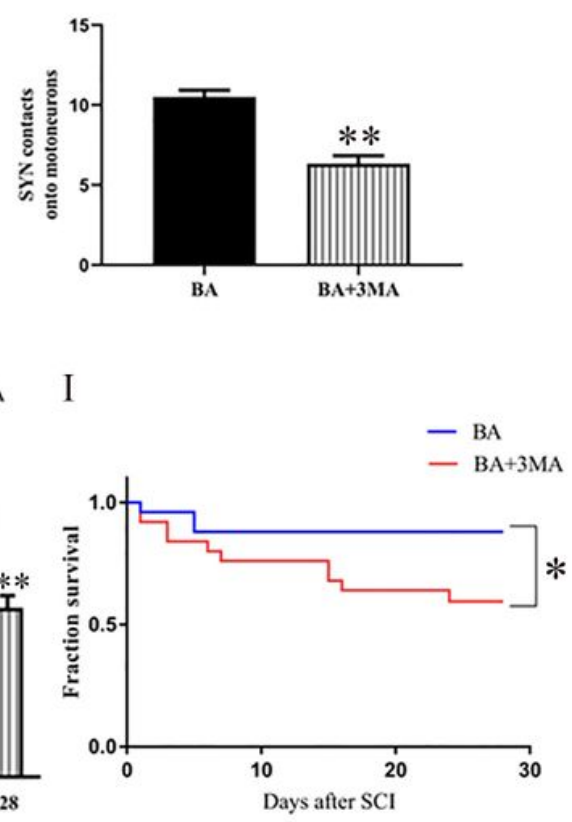


\section{Figure 5}

Inhibition of autophagy reverses the effects of BA on functional recovery after $\mathrm{SCl}$. (A) Longitudinal spinal cord sections from the indicated groups at day 14 were analyzed by HE staining and Masson

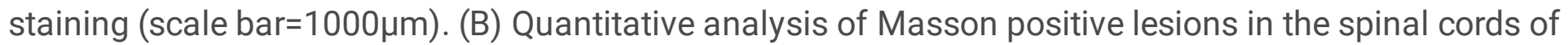
each group. (C) Images (30x) of the spinal cord sections in each group stained with antibodies against

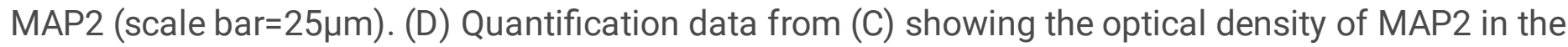
injured spinal cord at day 28. (E) Images (150x) of spinal cord sections below the injury (T11-T12) stained at day 28 with antibodies against SYN/NeuN (scale bar=5 $\mu \mathrm{m}$ ). (F) Corresponding quantification of the number of synapses contacting motor neurons. (G) Photos of mice footprints at day 28 after SCl. (H) Basso mouse scale (BMS) for the indicated groups and time points, $n=(6-12)$. (I) The analysis of survival rate of $B A$ mice and $B A+3 M A$ mice subjected to $S C l$ at days $1,7,14,21$, and 28 . The values are expressed as the means \pm SEM, $n=6$ per group (except for $B M S$ ). ${ }^{*} p<0.05$ and ${ }^{* \star} p<0.01$, vs. BA group. 

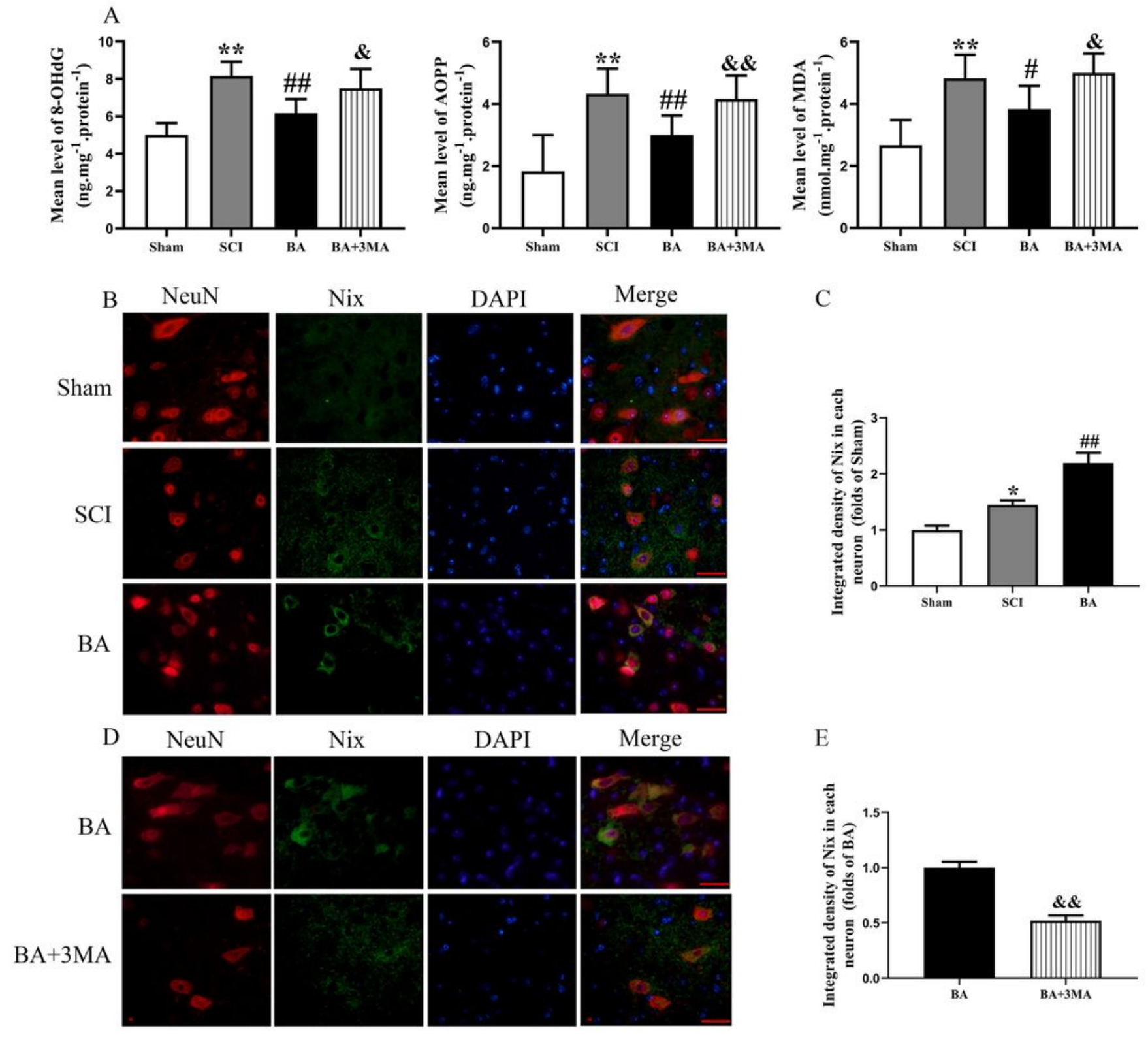

Merge

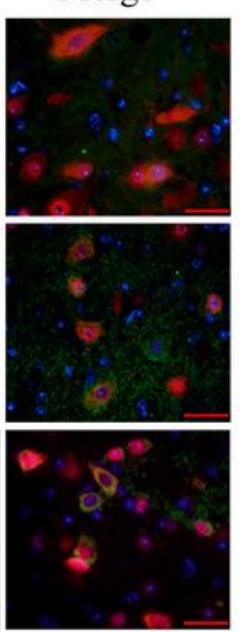

$\mathrm{C}$
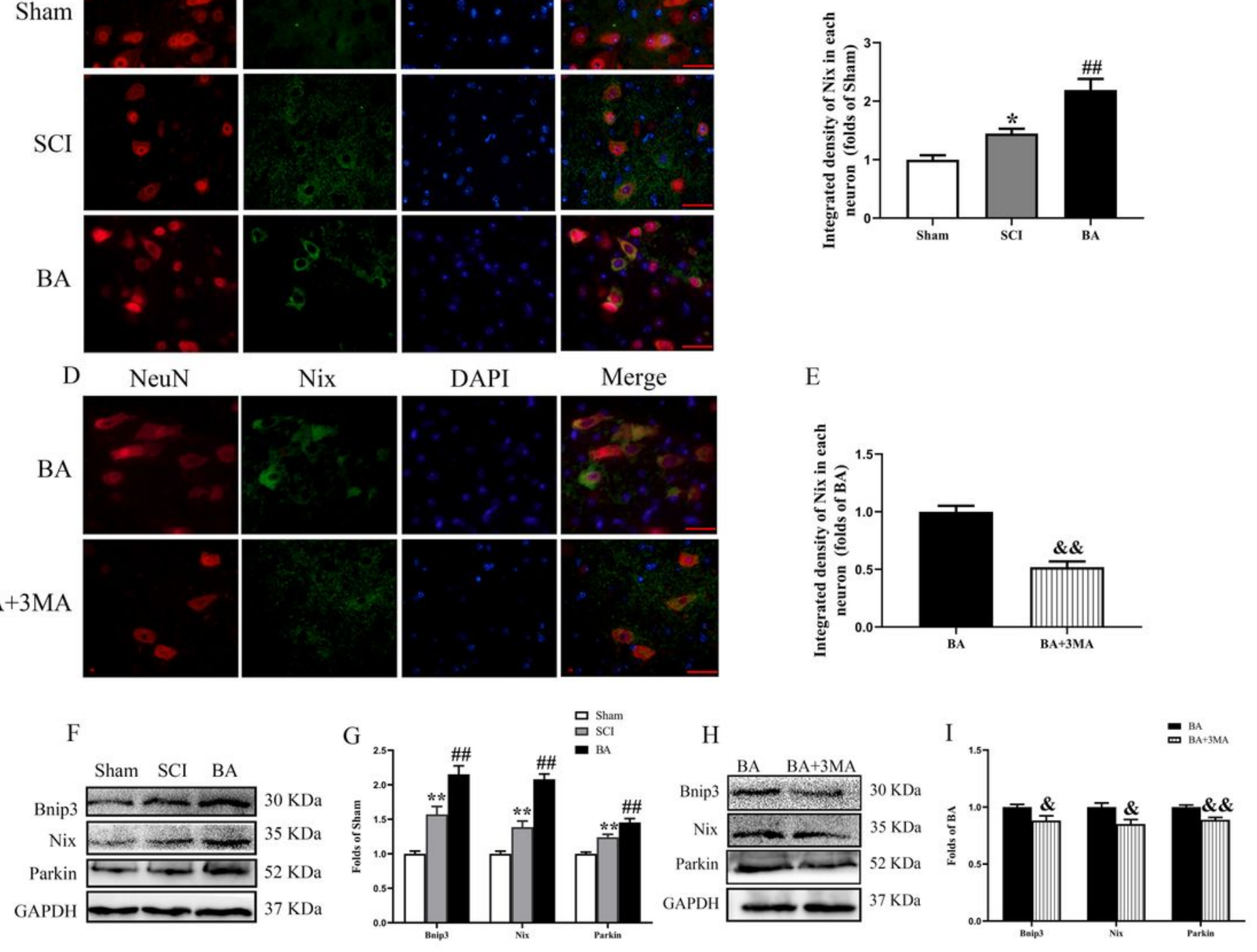

\section{Figure 6}

BA attenuates mitophagy and reduces ROS accumulation after SCI. (A) ELISA of 8-OHdG, AOPP, and MDA in spinal cord lesions from Sham, SCl, BA and BA+3MA groups as indicated. (B) Immunofluorescence staining for Nix and NeuN co-localization in the spinal cords of the Sham, $\mathrm{SCl}$, and BA groups (Scan bar $=25 \mu \mathrm{m})$. (C) The quantitative mean optical density of the Nix in motor neurons of spinal cord lesion in each group. (D) Immunofluorescence staining for Nix and NeuN co-localization in the spinal cords of the 


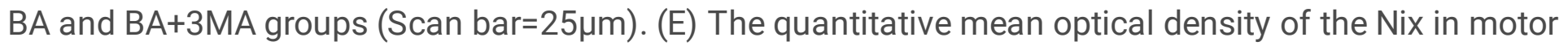
neurons of spinal cord lesion in each group. (F) Western blotting for Bnip3, Nix and Parkin expression levels in the Sham, $\mathrm{SCl}$ and $\mathrm{BA}$ groups. The gels were run under the same experimental conditions, and the cropped blots are shown here. $(G)$ The optical density values of the Bnip3, Nix and Parkin expression levels were quantified and analyzed in each group. $(\mathrm{H})$ Western blotting for Bnip3, Nix and Parkin expression levels in the BA and BA+3MA groups. The gels were run under the same experimental conditions, and the cropped blots are shown here. (I) The optical density values of the Bnip3, Nix and Parkin expression levels were quantified and analyzed in each group. The values are expressed as the means $\pm S E M, n=6$ per group. ${ }^{*} p<0.05$ and ${ }^{* \star} p<0.01$, vs. Sham group. $\# p<0.05$ and $\# \# p<0.01$, vs. $S C l$ group. $\& p<0.05$ and $\& \& p<0.01$, vs. BA group. 

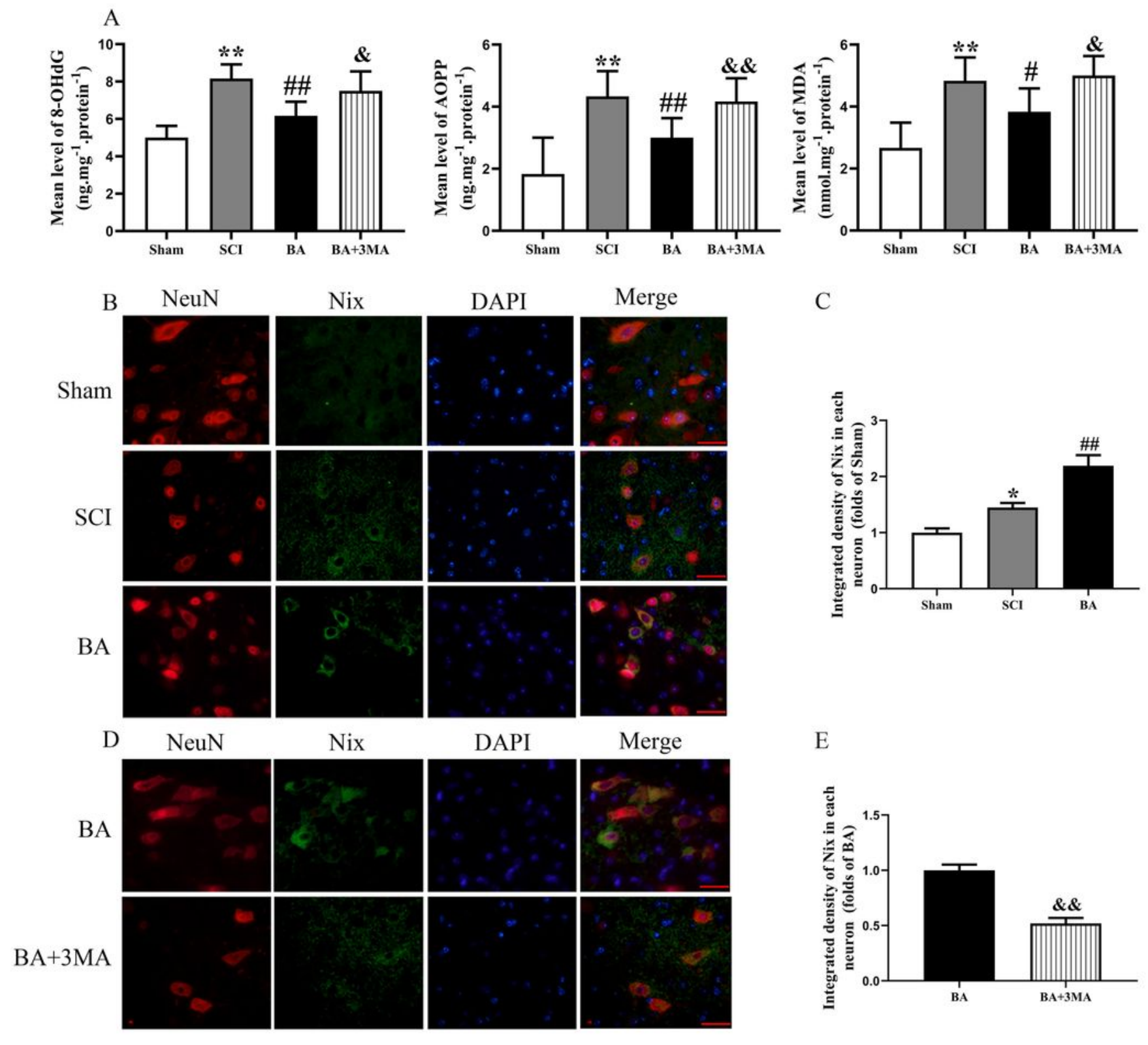

DAPI

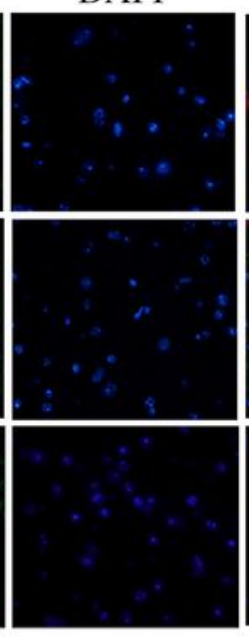

Merge

DAPI

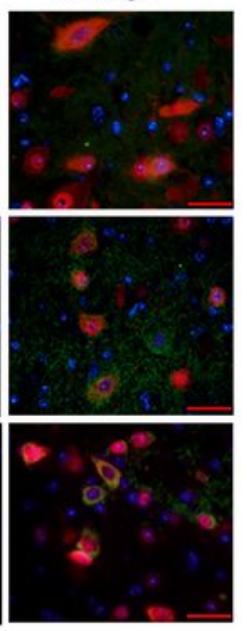

$\mathrm{C}$

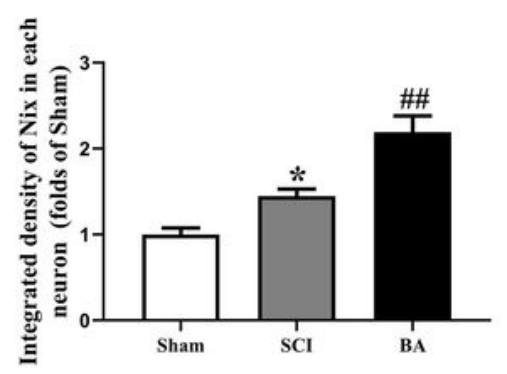

$\mathrm{E}$

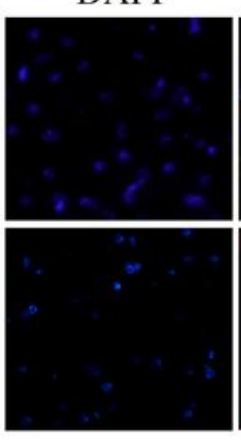

Merge
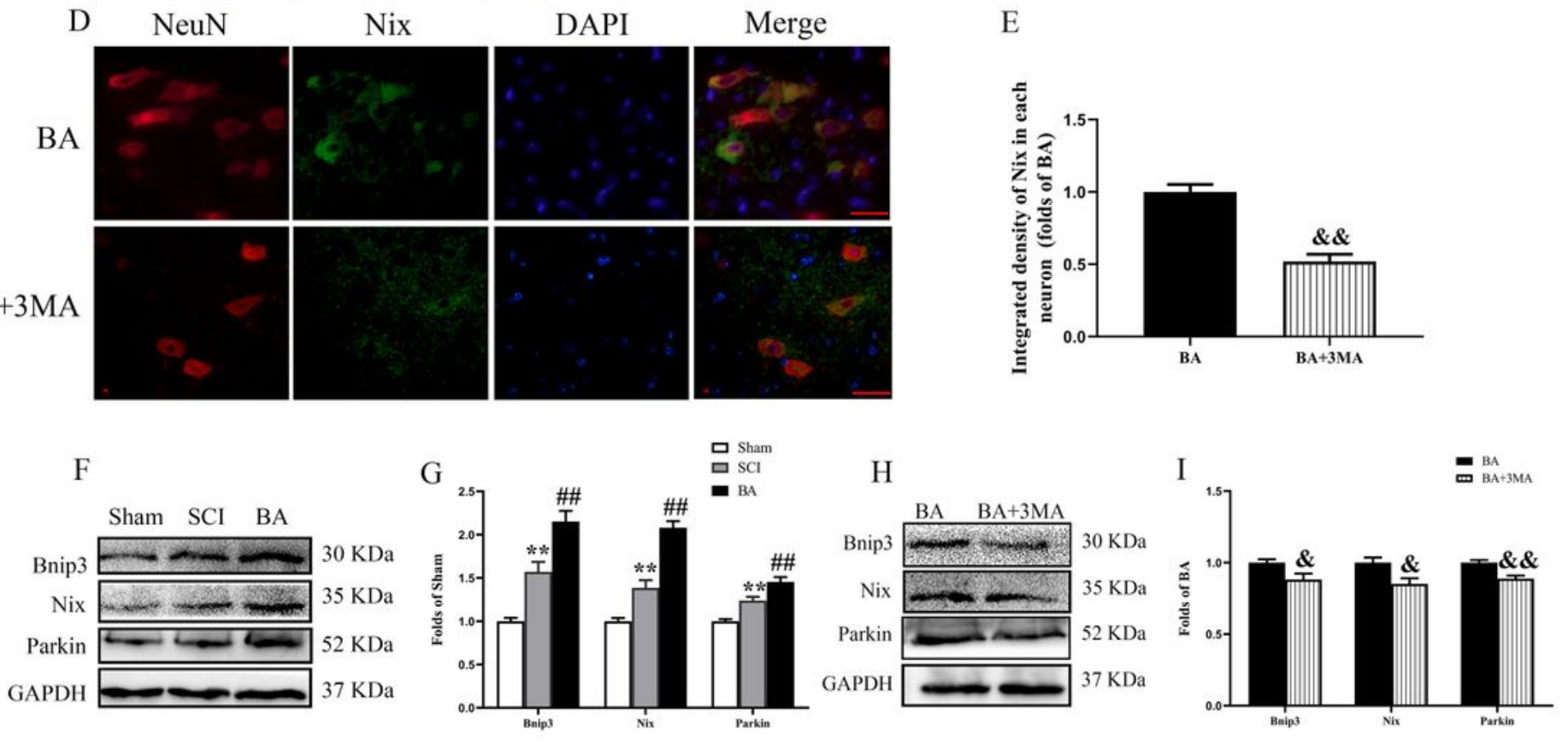

\section{Figure 6}

BA attenuates mitophagy and reduces ROS accumulation after SCI. (A) ELISA of 8-OHdG, AOPP, and MDA in spinal cord lesions from Sham, SCl, BA and BA+3MA groups as indicated. (B) Immunofluorescence staining for Nix and NeuN co-localization in the spinal cords of the Sham, $\mathrm{SCl}$, and $\mathrm{BA}$ groups (Scan

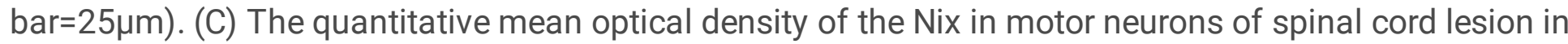
each group. (D) Immunofluorescence staining for Nix and NeuN co-localization in the spinal cords of the 


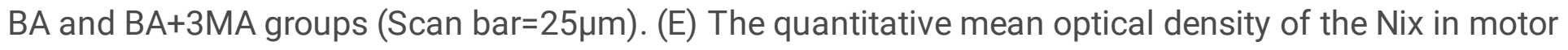
neurons of spinal cord lesion in each group. (F) Western blotting for Bnip3, Nix and Parkin expression levels in the Sham, $\mathrm{SCl}$ and $\mathrm{BA}$ groups. The gels were run under the same experimental conditions, and the cropped blots are shown here. $(G)$ The optical density values of the Bnip3, Nix and Parkin expression levels were quantified and analyzed in each group. $(\mathrm{H})$ Western blotting for Bnip3, Nix and Parkin expression levels in the BA and BA+3MA groups. The gels were run under the same experimental conditions, and the cropped blots are shown here. (I) The optical density values of the Bnip3, Nix and Parkin expression levels were quantified and analyzed in each group. The values are expressed as the means $\pm S E M, n=6$ per group. ${ }^{*} p<0.05$ and ${ }^{* \star} p<0.01$, vs. Sham group. $\# p<0.05$ and $\# \# p<0.01$, vs. $S C l$ group. $\& p<0.05$ and $\& \& p<0.01$, vs. BA group. 

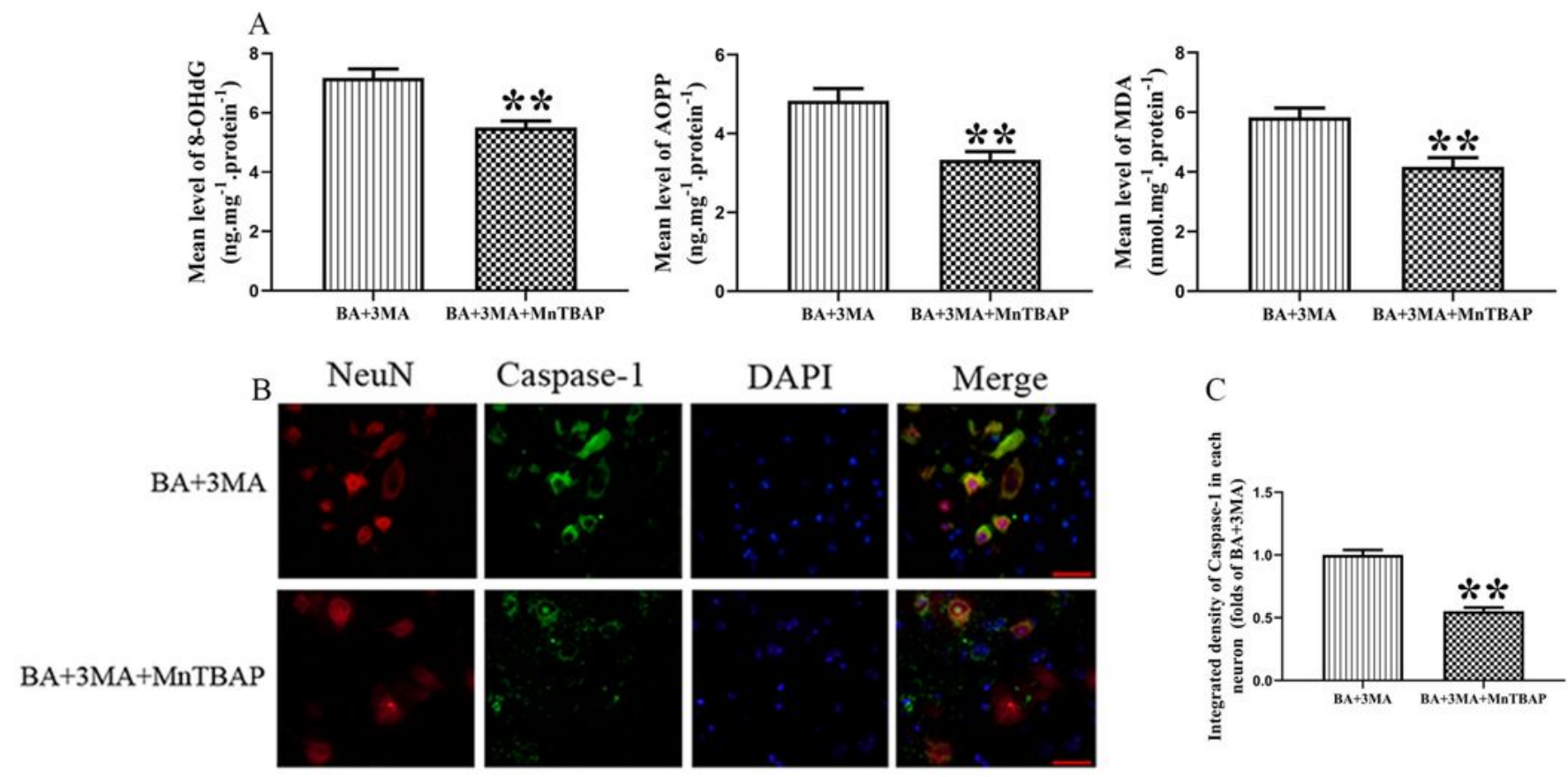

Caspase-1

DAPI
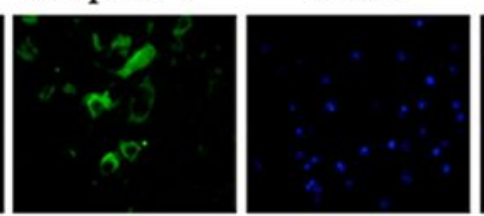

Merge
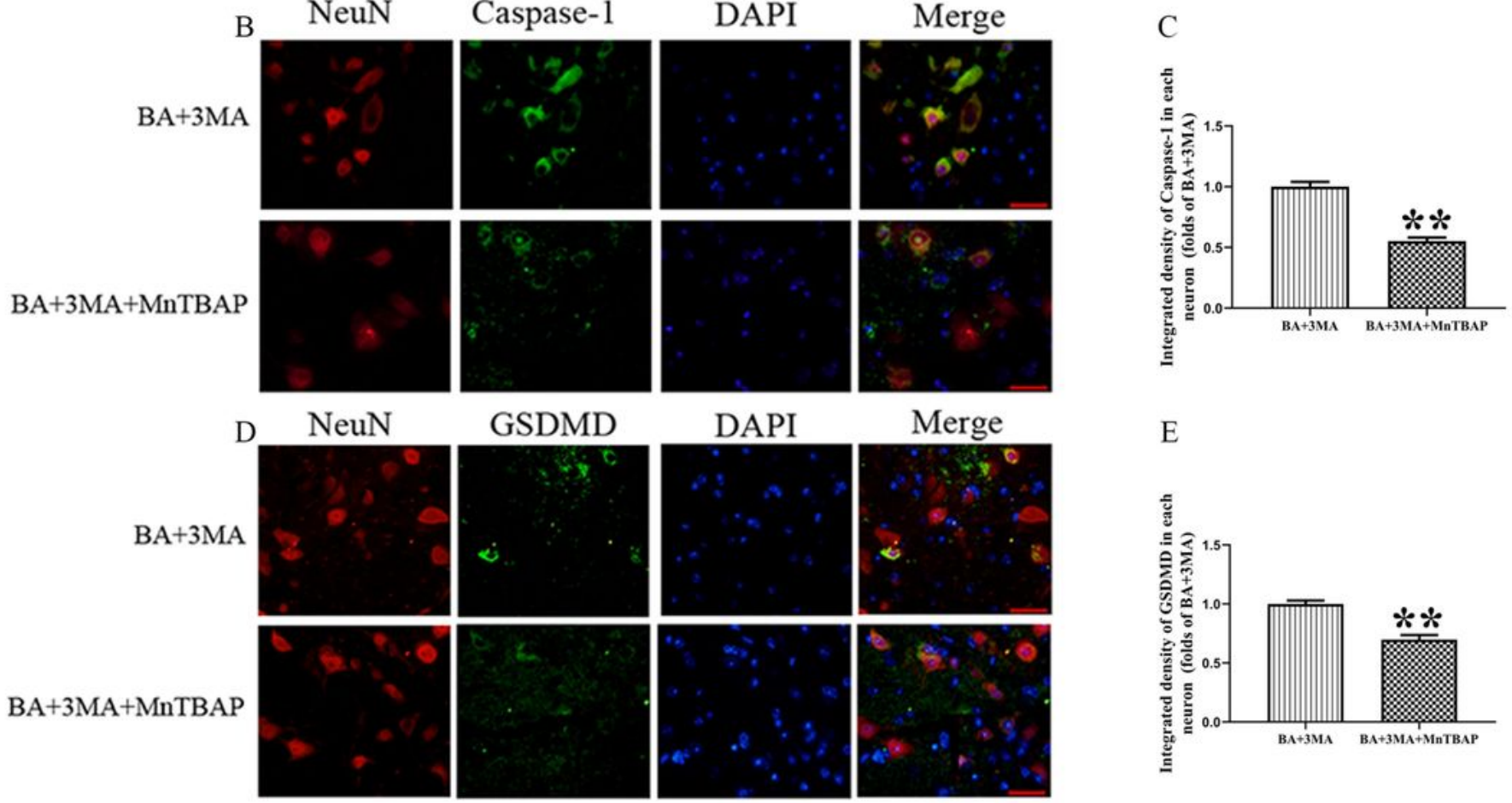

DAPI
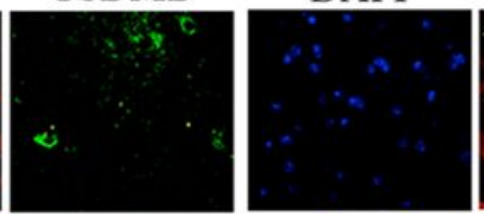

Merge

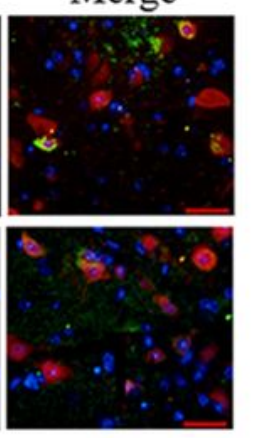

E
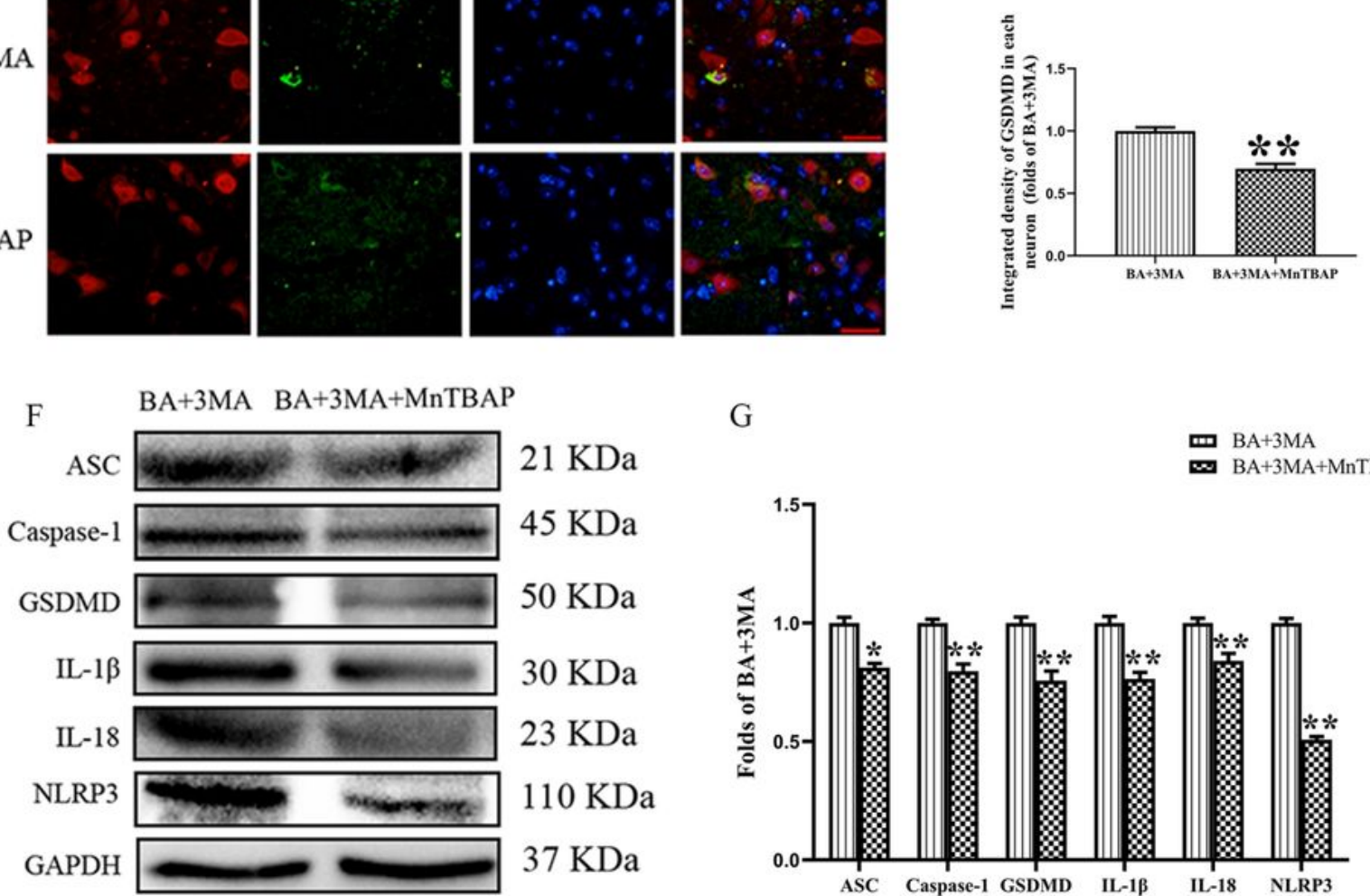

$21 \mathrm{KDa}$

G $45 \mathrm{KDa}$

$50 \mathrm{KDa}$

$30 \mathrm{KDa}$

$23 \mathrm{KDa}$

$110 \mathrm{KDa}$

$37 \mathrm{KDa}$

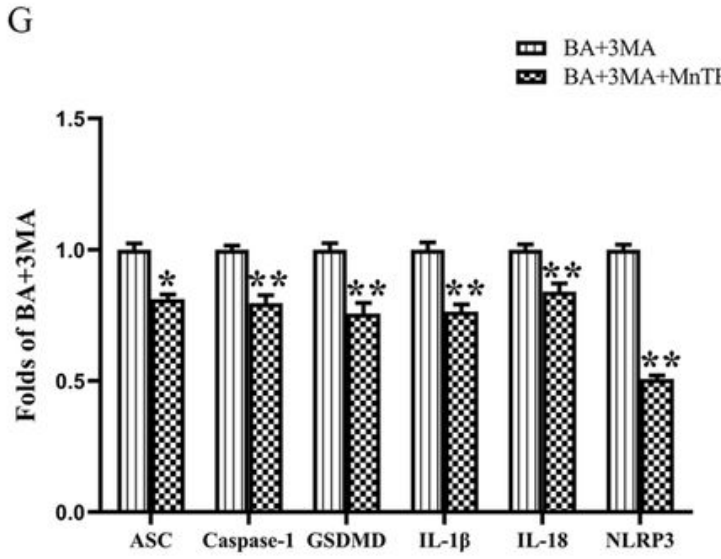

\section{Figure 7}

BA inhibits pyroptosis via mitophagy induced ROS elimination. (A) ELISA of 8-OHdG, AOPP, and MDA in spinal cord lesions from $B A+3 M A$ and $B A+3 M A+M n T B A P$ groups as indicated. (B) Immunofluorescence staining for Caspase-1 and NeuN co-localization in the spinal cords of the BA+3MA and $B A+3 M A+M n T B A P$ groups (Scan bar=25 $\mu \mathrm{m}$ ). (C) The quantitative mean optical density of the Caspase-1 in motor neurons of spinal cord lesion in each group. (D) Immunofluorescence staining for GSDMD and 
NeuN co-localization in the spinal cords of the BA+3MA and BA+3MA+MnTBAP groups (Scan bar $=25 \mu \mathrm{m}) .(E)$ The quantitative mean optical density of the GSDMD in motor neurons of spinal cord lesion in each group. (F) Western blotting for ASC, Caspase-1, GSDMD, IL-1 $\beta$, IL-18 and NLRP3 expression levels in the BA+3MA and BA+3MA+MnTBAP groups. The gels were run under the same experimental conditions, and the cropped blots are shown here. (G) The optical density values of the ASC, Caspase-1, GSDMD, IL-1 $\beta$, IL-18 and NLRP3 expression levels were quantified and analyzed in each group. The values are expressed as the means $\pm S E M, n=6$ per group. ${ }^{*} p<0.05$ and ${ }^{* *} p<0.01$, vs. BA+3MA group.
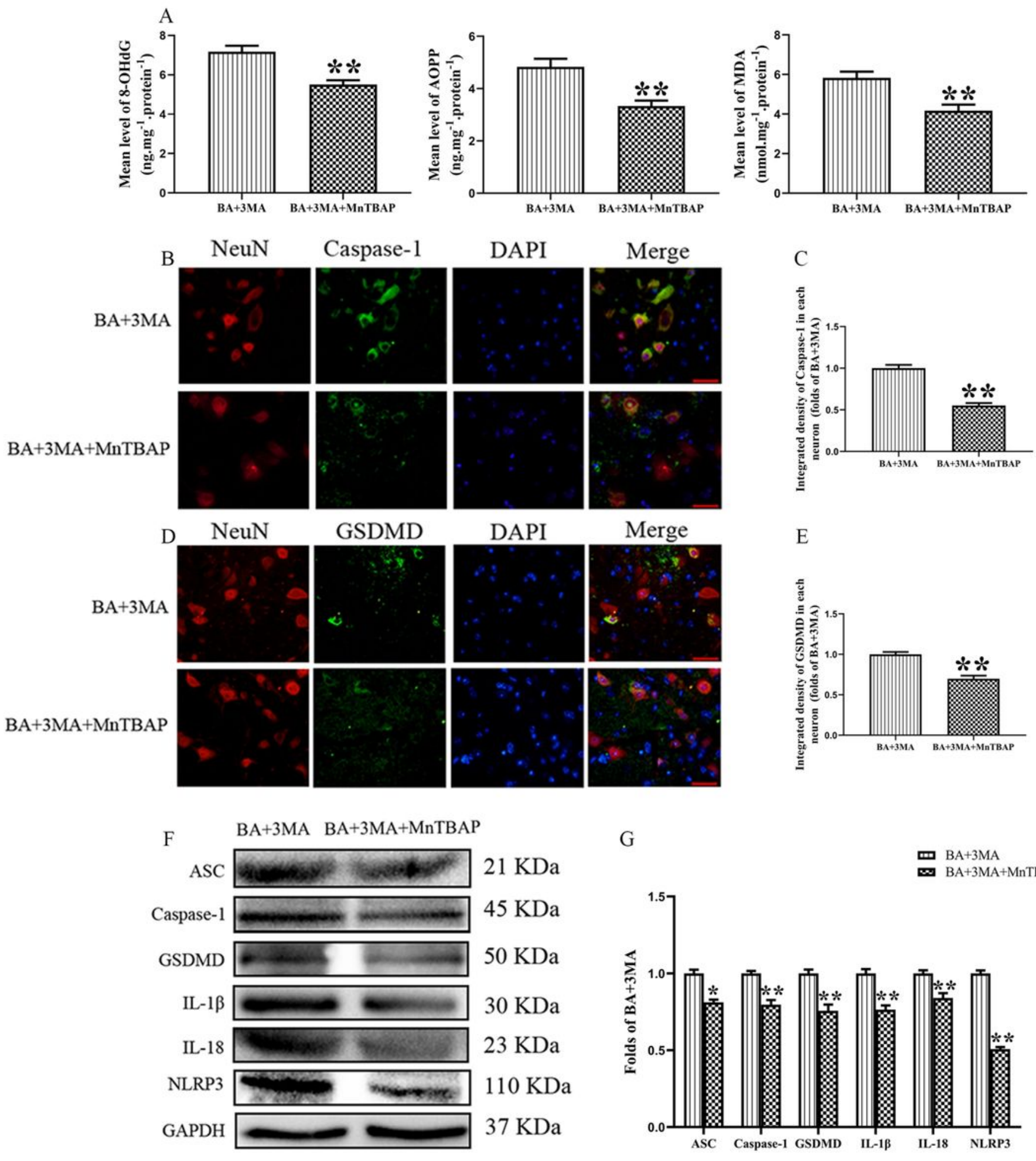

G $21 \mathrm{KDa}$ $45 \mathrm{KDa}$ $50 \mathrm{KDa}$ $30 \mathrm{KDa}$ $23 \mathrm{KDa}$ $110 \mathrm{KDa}$ $37 \mathrm{KDa}$
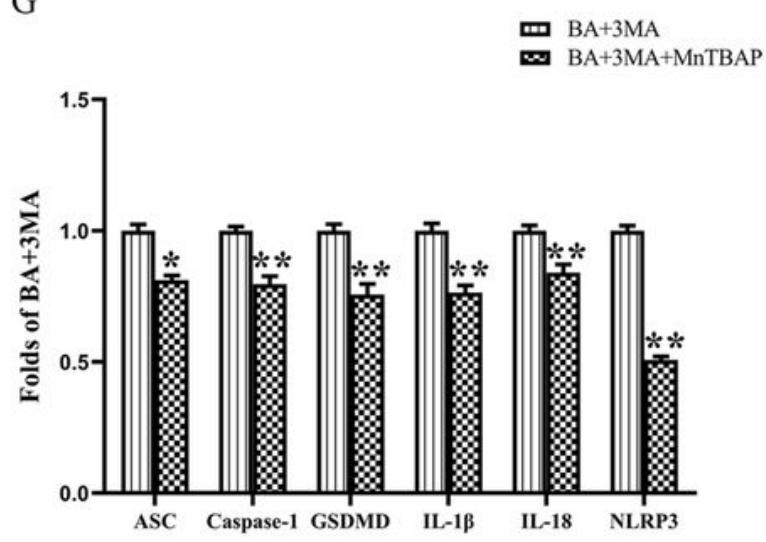


\section{Figure 7}

BA inhibits pyroptosis via mitophagy induced ROS elimination. (A) ELISA of 8-OHdG, AOPP, and MDA in spinal cord lesions from $B A+3 M A$ and $B A+3 M A+M n T B A P$ groups as indicated. (B) Immunofluorescence staining for Caspase-1 and NeuN co-localization in the spinal cords of the BA+3MA and BA+3MA+MnTBAP groups (Scan bar=25 $\mu \mathrm{m}$ ). (C) The quantitative mean optical density of the Caspase-1 in motor neurons of spinal cord lesion in each group. (D) Immunofluorescence staining for GSDMD and NeuN co-localization in the spinal cords of the BA+3MA and BA+3MA+MnTBAP groups (Scan bar $=25 \mu \mathrm{m}) .(E)$ The quantitative mean optical density of the GSDMD in motor neurons of spinal cord lesion in each group. (F) Western blotting for ASC, Caspase-1, GSDMD, IL-1 $\beta$, IL-18 and NLRP3 expression levels in the $B A+3 M A$ and $B A+3 M A+M n T B A P$ groups. The gels were run under the same experimental conditions, and the cropped blots are shown here. (G) The optical density values of the ASC, Caspase-1, GSDMD, IL-1 $\beta$, IL-18 and NLRP3 expression levels were quantified and analyzed in each group. The values are expressed as the means $\pm S E M, n=6$ per group. ${ }^{*} p<0.05$ and ${ }^{* \star} p<0.01$, vs. BA+3MA group. 
A

B

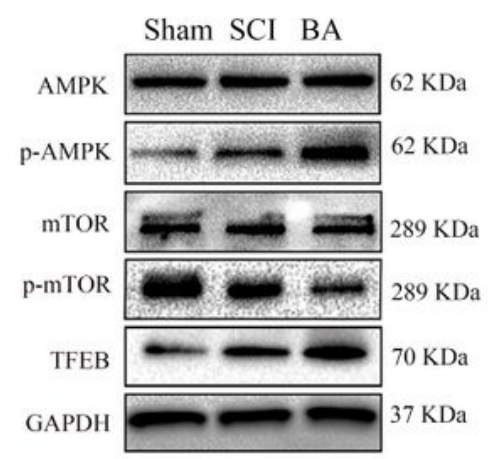

D

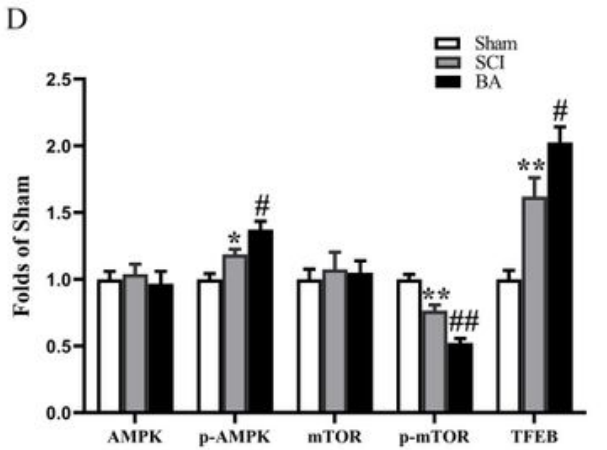

$\mathrm{E}$

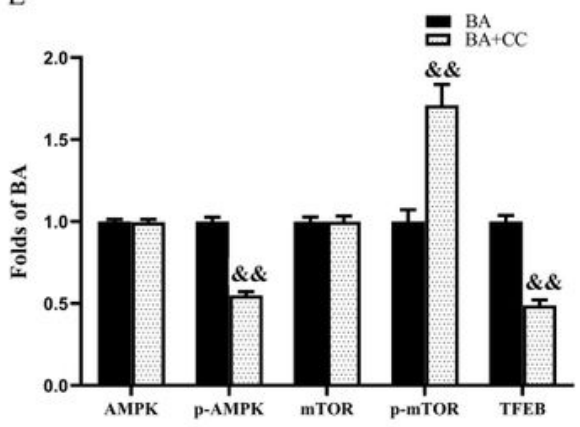

C

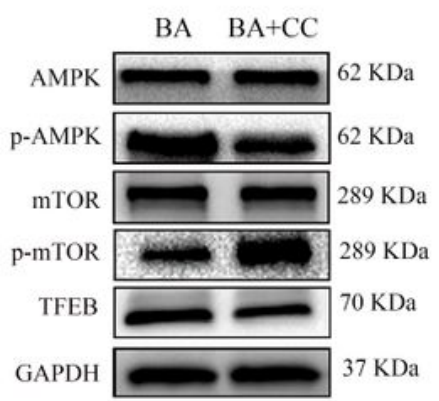

F

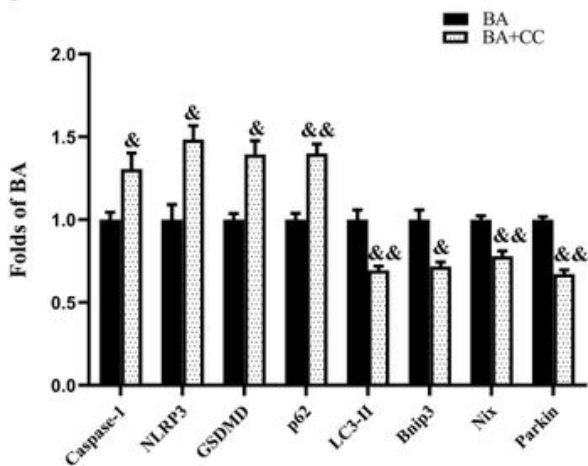

G

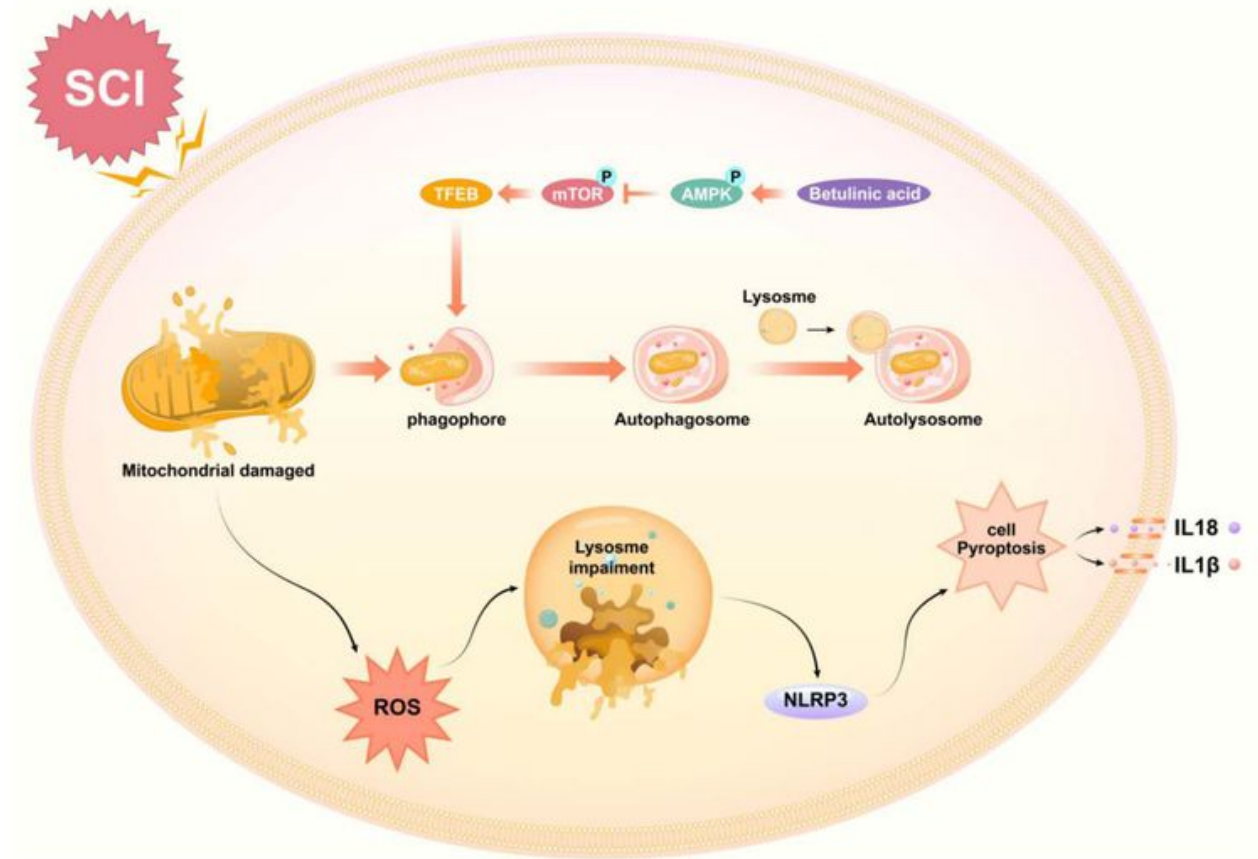

\section{Figure 8}

BA activates autophagy and mitophagy via enhancing AMPK-mTOR -TFEB activity. (A) Western blotting for AMPK, p-AMPK, mTOR, p-mTOR and TFEB expression levels in the Sham, SCl and BA groups. The gels were run under the same experimental conditions, and the cropped blots are shown here. (D) The optical density values of the AMPK, p-AMPK, mTOR, p-mTOR and TFEB expression levels were quantified and analyzed in each group. (B) Western blotting for AMPK, p-AMPK, mTOR, p-mTOR and TFEB expression 
levels in the BA and BA+3MA groups. The gels were run under the same experimental conditions, and the cropped blots are shown here. (E) The optical density values of the AMPK, p-AMPK, mTOR, p-mTOR and TFEB expression levels were quantified and analyzed in each group. (C) Western blotting for Caspase-1, NLRP3, GSDMD, p62, LC3II, Bnip3, Nix and Parkin expression levels in the BA and BA+CC groups. The gels were run under the same experimental conditions, and the cropped blots are shown here. (F) The optical density values of the Caspase-1, NLRP3, GSDMD, p62, LC3II, Bnip3, Nix and Parkin expression levels were quantified and analyzed in each group. (G) Schematic illustration of the proposed molecular mechanism highlighting the role of betulinic acid in the pathophysiology of $\mathrm{SCl}$ and subsequent neurological recovery. Spinal cord injury results in the mitochondrial damage which leads to a massive accumulation of ROS and subsequently to induce pyroptosis. Betulinic acid activates autophagy through AMPK-mTORTFEB signaling pathway. Then enhanced autophagy further induces mitophagy to contribute to the elimination of damaged mitochondria and ROS by forming autolysosomes with lysosomes.

Subsequently, pyroptosis are inhibited, which further results in the promotion of functional recovery. The values are expressed as the means $\pm S E M, n=6$ per group. ${ }^{*} p<0.05$ and ${ }^{* \star} p<0.01$, vs. Sham group. \#p< 0.05 and $\# \# p<0.01$, vs. SCl group. $\& p<0.05$ and $\& \& p<0.01$, vs. BA group. 
B
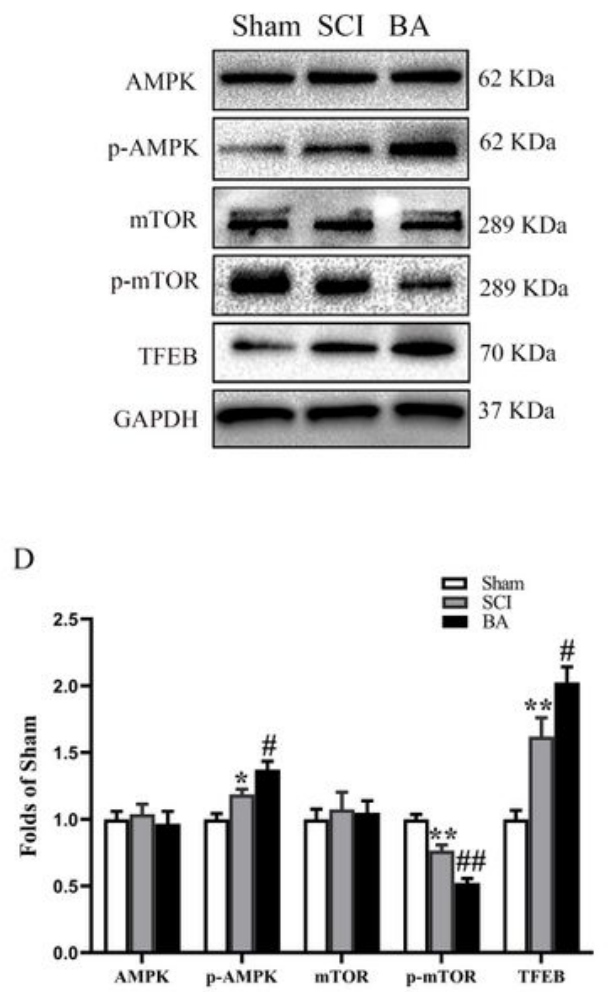

$\mathrm{E}$
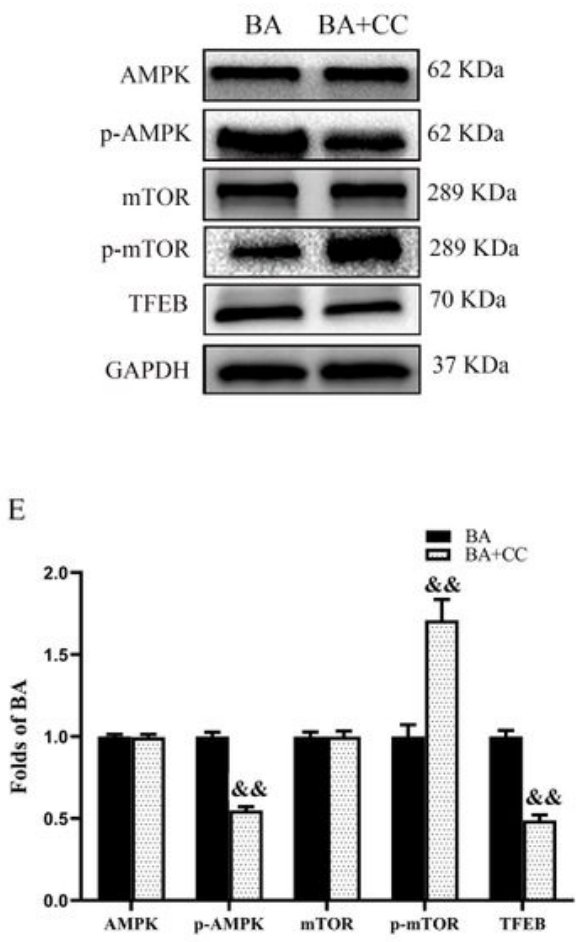

C

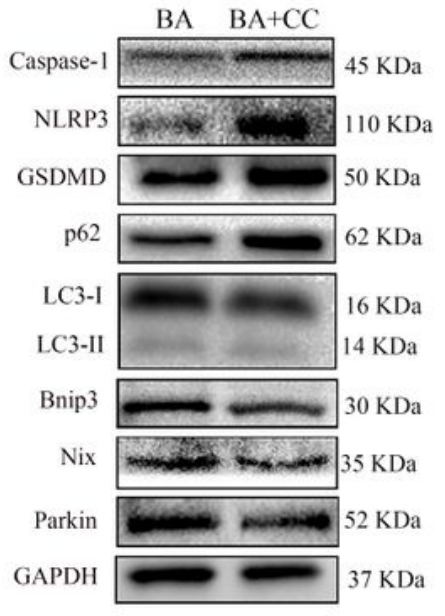

$\mathrm{F}$

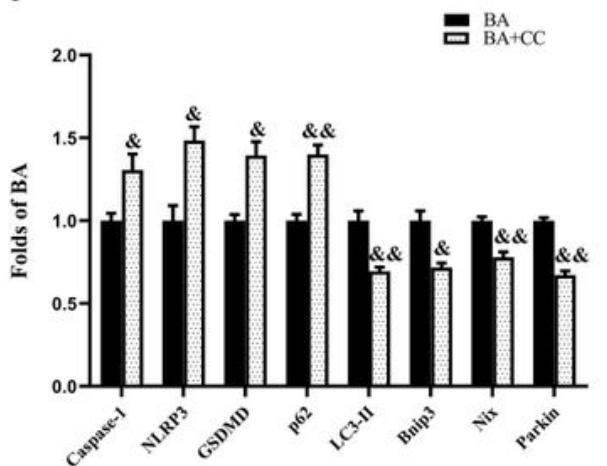

G

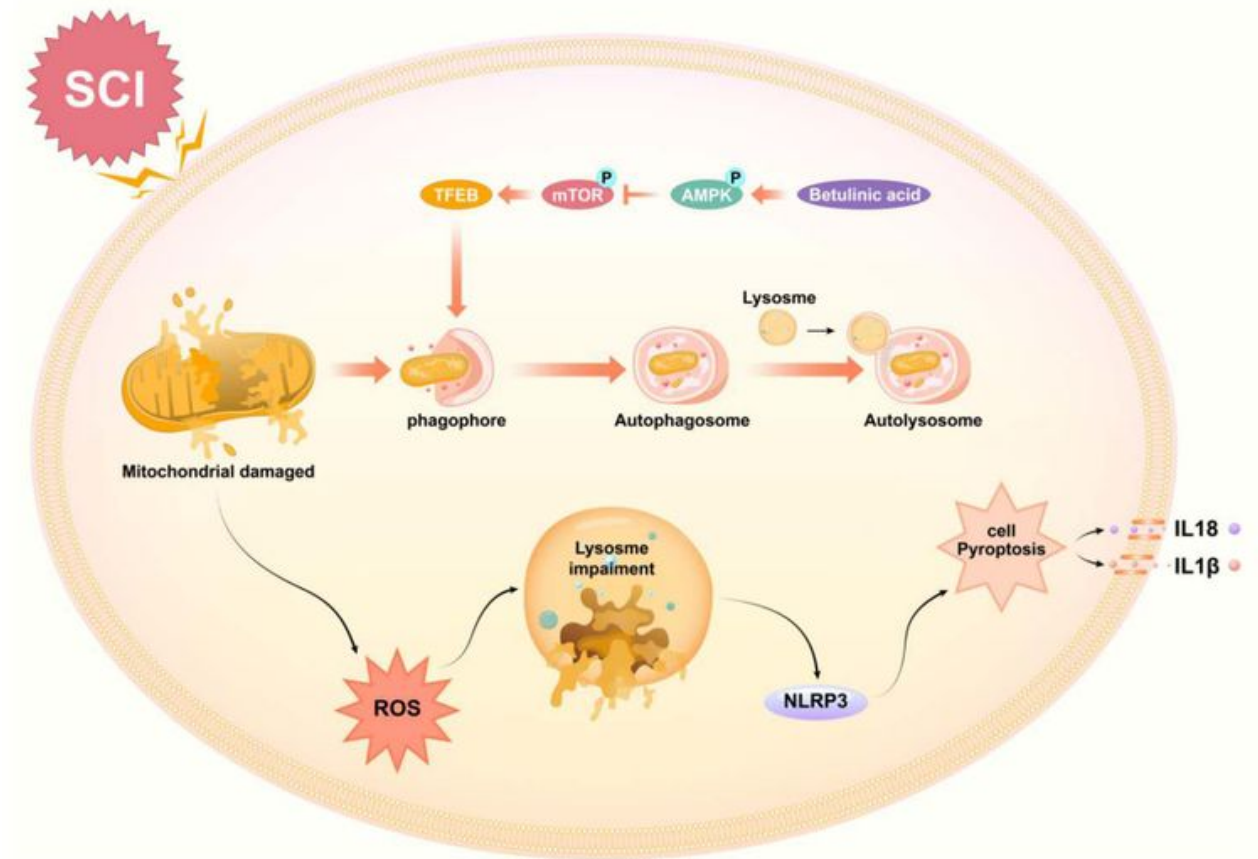

\section{Figure 8}

BA activates autophagy and mitophagy via enhancing AMPK-mTOR -TFEB activity. (A) Western blotting for AMPK, p-AMPK, mTOR, p-mTOR and TFEB expression levels in the Sham, SCl and BA groups. The gels were run under the same experimental conditions, and the cropped blots are shown here. (D) The optical density values of the AMPK, p-AMPK, mTOR, p-mTOR and TFEB expression levels were quantified and analyzed in each group. (B) Western blotting for AMPK, p-AMPK, mTOR, p-mTOR and TFEB expression 
levels in the BA and BA+3MA groups. The gels were run under the same experimental conditions, and the cropped blots are shown here. (E) The optical density values of the AMPK, p-AMPK, mTOR, p-mTOR and TFEB expression levels were quantified and analyzed in each group. (C) Western blotting for Caspase-1, NLRP3, GSDMD, p62, LC3II, Bnip3, Nix and Parkin expression levels in the BA and BA+CC groups. The gels were run under the same experimental conditions, and the cropped blots are shown here. (F) The optical density values of the Caspase-1, NLRP3, GSDMD, p62, LC3II, Bnip3, Nix and Parkin expression levels were quantified and analyzed in each group. (G) Schematic illustration of the proposed molecular mechanism highlighting the role of betulinic acid in the pathophysiology of $\mathrm{SCl}$ and subsequent neurological recovery. Spinal cord injury results in the mitochondrial damage which leads to a massive accumulation of ROS and subsequently to induce pyroptosis. Betulinic acid activates autophagy through AMPK-mTORTFEB signaling pathway. Then enhanced autophagy further induces mitophagy to contribute to the elimination of damaged mitochondria and ROS by forming autolysosomes with lysosomes.

Subsequently, pyroptosis are inhibited, which further results in the promotion of functional recovery. The values are expressed as the means $\pm S E M, n=6$ per group. ${ }^{*} p<0.05$ and ${ }^{* \star} p<0.01$, vs. Sham group. \#p< 0.05 and $\# \# p<0.01$, vs. SCl group. $\& p<0.05$ and $\& \& p<0.01$, vs. BA group. 